\title{
Ventcel' boundary value problems for elliptic Waldenfels operators
}

\section{Kazuaki Taira}

Dedicated to Professor Silvia Romanelli on the occasion of her 70th birthday

Received: date / Accepted: date

\begin{abstract}
In this paper we study a class of first-order Ventcel' boundary value problems for second-order, elliptic Waldenfels integro-differential operators. More precisely, by using real analysis techniques such as Strichartz norms and the complex interpolation method we prove existence and uniqueness theorems in the framework of Sobolev and Besov spaces of $L^{p}$ type which extend earlier theorems due to Bony-Courrège-Priouret and Runst-Youssfi to the general degenerate case. Our proof is based on various maximum principles for second-order, elliptic Waldenfels operators with discontinuous coefficients in the framework of $L^{p}$ Sobolev spaces.
\end{abstract}

Keywords Waldenfels integral-differential operator · Ventcel' (Wentzell) boundary condition $\cdot$ Sobolev space $\cdot$ Besov space $\cdot$ Strichartz norm $\cdot$ complex interpolation method $\cdot$ maximum principle

Mathematics Subject Classification (2010) 35J25 - 47G10 - 47G20 • $46 \mathrm{E} 35 \cdot 47 \mathrm{D} 07 \cdot 47 \mathrm{H} 30$

\section{Contents}

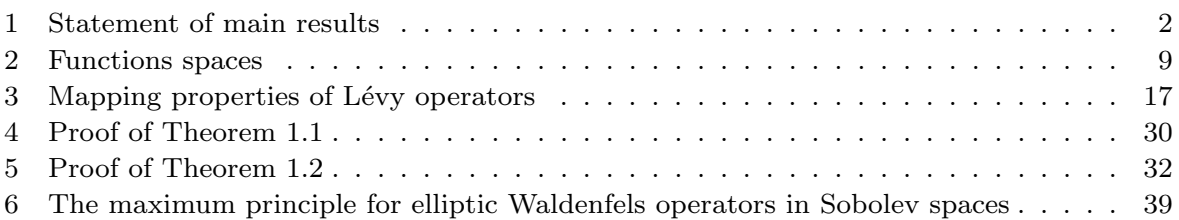




\section{Statement of main results}

Let $\Omega$ be a bounded domain of Euclidean space $\mathbf{R}^{n}, n \geq 2$, with smooth boundary $\partial \Omega$; its closure $\bar{\Omega}=\Omega \cup \partial \Omega$ is an $n$-dimensional, compact smooth manifold with boundary.

First, we consider a second-order, uniformly elliptic integro-differential operator $W$ with real coefficients such that

$$
W u(x)=A u(x)+S u(x) \text { for } x \in \Omega,
$$

where $A$ is a second-order, differential operator given by the formula

$$
A u(x)=\sum_{i, j=1}^{n} a^{i j}(x) \frac{\partial^{2} u}{\partial x_{i} \partial x_{j}}(x)+\sum_{i=1}^{n} b^{i}(x) \frac{\partial u}{\partial x_{i}}(x)+c(x) u(x)
$$

and $S$ is a second-order, integro-differential operator given by the formula

$$
S u(x)=\int_{\mathbf{R}^{n} \backslash\{0\}}\left(u(x+z)-u(x)-\sum_{k=1}^{n} z_{k} \frac{\partial u}{\partial x_{k}}(x)\right) K(x, z) \mu(d z) .
$$

Here:

(1) $a^{i j} \in C^{\infty}(\bar{\Omega})$ and $a^{i j}(x)=a^{j i}(x)$ for all $x \in \bar{\Omega}$ and $1 \leq i, j \leq n$, and there exists a constant $a_{0}>0$ such that

$$
\sum_{i, j=1}^{n} a^{i j}(x) \xi_{i} \xi_{j} \geq a_{0}|\xi|^{2} \quad \text { for all }(x, \xi) \in \bar{\Omega} \times \mathbf{R}^{n} .
$$

(2) $b^{i} \in C^{\infty}(\bar{\Omega})$ for all $1 \leq i \leq n$.

(3) $c \in C^{\infty}(\bar{\Omega})$, and $c(x) \leq 0$ in $\Omega$, but $c(x) \not \equiv 0$ in $\Omega$.

(4) $K \in L^{\infty}\left(\mathbf{R}^{n} \times \mathbf{R}^{n}\right)$ with $K(x, y) \geq 0$ almost everywhere in $\mathbf{R}^{n} \times \mathbf{R}^{n}$, and satisfies the growth condition

$$
\begin{aligned}
H\left(\theta_{0}\right):= & \sup _{x, z \in \mathbf{R}^{n}} \int_{\mathbf{R}^{n}}|K(x+h, z)-K(x, z)|^{2} \frac{d h}{|h|^{n+2 \theta_{0}}}<\infty \\
& \quad \text { or some } 0<\theta_{0}<1
\end{aligned}
$$

and the support condition

$$
K(x, z)=0 \quad \text { if } x \in \Omega \text { and } x+z \notin \bar{\Omega} .
$$

The support condition (1.5) implies that all jumps from $\Omega$ are within $\bar{\Omega}$. The function $K(x, z)$ is called the jump density (see [12, Chapter 2]).

(5) $\mu(d z)$ is a Radon measure on $\mathbf{R}^{n} \backslash\{0\}$ which has a density with respect to the Lebesgue measure $d z$ on $\mathbf{R}^{n}$, and satisfies the moment condition ([19])

$$
\int_{\{0<|z| \leq 1\}}|z|^{2} \mu(d z)+\int_{\{|z|>1\}}|z| \mu(d z)<\infty .
$$


The moment condition (1.6) implies that the measure $\mu(\cdot)$ admits a singularity of order 2 at the origin, and this singularity at the origin is produced by the accumulation of small jumps of Markovian particles, while the measure $\mu(\cdot)$ admits a singularity of order 1 at infinity, and this singularity at infinity is produced by the accumulation of large jumps of Markovian particles. The Radon measure $K(x, z) \mu(d z)$ is called the Lévy kernel.

Example 1.1 A typical example of the Radon measure $\mu(d z)$ which satisfies the moment condition (1.6) is given by the formula

$$
\mu(d z)= \begin{cases}\frac{1}{|z|^{n+2-\varepsilon}} d z & \text { for } 0<|z| \leq 1 \\ \frac{1}{|z|^{n+1+\varepsilon}} d z & \text { for }|z|>1\end{cases}
$$

where $\varepsilon>0$.

Note that condition (1.5) guarantees that the operator $S$ can be interpreted as a mapping acting on functions $u$ which are defined in $\bar{\Omega}$. In this sense, the condition $H\left(\theta_{0}\right)<\infty$ should be considered as a condition with respect to the closure $\bar{\Omega}$.

The operator $W$ is called a second-order, Waldenfels integro-differential operator (cf. [40], [7]). The operator $A$ is called a diffusion operator which describes analytically a strong Markov process with continuous paths (diffusion process) in the interior $\Omega([32])$. In fact, we remark that the differential operator $A$ is local, that is, the value $A u\left(x^{0}\right)$ at an interior point $x^{0} \in \Omega$ is determined by the values of $u$ in an arbitrary small neighborhood of $x^{0}$. Moreover, it is known from Peetre's theorem ([21]) that a linear operator is local if and only if it is a differential operator. The operator $S$ is called a secondorder, Lévy integro-differential operator which is supposed to correspond to the jump phenomenon in the interior $\Omega$; a Markovian particle moves by jumps to a random point, chosen with kernel $K(x, z)$, in the interior $\Omega([18],[29],[25]$, $[17])$. Therefore, the Waldenfels integro-differential operator $W$ is supposed to correspond to such a diffusion phenomenon that a Markovian particle moves both by jumps and continuously in the state space $\Omega$.

Secondly, we consider a first-order, boundary operator $L$ with real coefficients such that

$$
L u\left(x^{\prime}\right)=\Lambda u\left(x^{\prime}\right)+\gamma_{0}(T u)\left(x^{\prime}\right) \quad \text { for } x^{\prime} \in \partial \Omega,
$$

where $\Lambda u$ is a first-order boundary condition given by the formula

$$
\Lambda u\left(x^{\prime}\right)=\mu\left(x^{\prime}\right) \frac{\partial u}{\partial \mathbf{n}}\left(x^{\prime}\right)+\gamma\left(x^{\prime}\right) u\left(x^{\prime}\right),
$$

and $\gamma_{0}(T u)$ is a first-order, integral operator defined by the formula

$$
\gamma_{0}(T u)\left(x^{\prime}\right)=T u\left(x^{\prime}\right)=\int_{\mathbf{R}^{n} \backslash\{0\}}\left(u\left(x^{\prime}+z\right)-u\left(x^{\prime}\right)\right) J\left(x^{\prime}, z\right) \nu(d z) .
$$

Here: 
(1) $\mu \in C^{\infty}(\partial \Omega)$ and $\mu\left(x^{\prime}\right) \geq 0$ on $\partial \Omega$.

(2) $\gamma \in C^{\infty}(\partial \Omega)$ and $\gamma\left(x^{\prime}\right) \leq 0$ on $\partial \Omega$.

(3) $\mathbf{n}=\left(n_{1}, n_{2}, \ldots, n_{n}\right)$ is the unit inward normal to the boundary $\partial \Omega$.

(4) $J \in L^{\infty}\left(\mathbf{R}^{n} \times \mathbf{R}^{n}\right)$ with $J(x, y) \geq 0$ almost everywhere in $\mathbf{R}^{n} \times \mathbf{R}^{n}$, and satisfies the support condition

$$
J(x, z)=0 \quad \text { if } x \in \bar{\Omega} \text { and } x+z \notin \bar{\Omega} .
$$

In view of formula (1.8), the support condition (1.9) implies that all jumps from $\partial \Omega$ are within $\bar{\Omega}$. Moreover, the function $J(x, z)$ satisfies the Lipschitz condition with respect to the variable $x$ for some $C_{1}>0$

$$
\begin{aligned}
& |J(x+h, z)-J(x, z)| \leq C_{1}|h| \\
& \quad \text { for all } x, h \in \mathbf{R}^{n} \text { and almost all } z \in \mathbf{R}^{n},
\end{aligned}
$$

and its weak partial derivatives $\partial J / \partial x_{i} \in L^{\infty}\left(\mathbf{R}^{n} \times \mathbf{R}^{n}\right), 1 \leq i \leq n$, satisfy the growth condition

$$
\begin{aligned}
G\left(\theta_{1}\right):= & \sup _{x, z \in \mathbf{R}^{n}} \int_{\mathbf{R}^{n}}\left|\nabla_{x} J(x+h, z)-\nabla_{x} J(x, z)\right|^{2} \frac{d h}{|h|^{n+2 \theta_{1}}}<\infty \\
& \quad \text { for some } 0<\theta_{1}<1 .
\end{aligned}
$$

(5) $\nu(d z)$ is a Radon measure on $\mathbf{R}^{n} \backslash\{0\}$ which has a density with respect to the Lebesgue measure $d z$ on $\mathbf{R}^{n}$, and satisfies the moment condition

$$
\int_{\{0<|z| \leq 1\}}|z| \nu(d z)+\int_{\{|z|>1\}} \nu(d z)<\infty .
$$

The moment condition (1.12) implies that the measure $\nu(\cdot)$ admits a singularity of order 1 at the origin, and this singularity at the origin is produced by the accumulation of small jumps of Markovian particles.

Example 1.2 A typical example of the Radon measure $\nu(d z)$ which satisfies the moment condition (1.12) is given by the formula

$$
\nu(d z)= \begin{cases}\frac{1}{|z|^{n+1-\varepsilon}} d z & \text { for } 0<|z| \leq 1 \\ \frac{1}{|z|^{n+\varepsilon}} d z & \text { for }|z|>1\end{cases}
$$

where $\varepsilon>0$.

Remark 1.1 It follows from Rademacher's theorem (see [20, Chapter 1, Corollary 1.73], [44, Theorem 2.2.1]) that any Lipschitz continuous function on $\mathbf{R}^{n}$ admits $L^{\infty}$ first partial derivatives almost everywhere in $\mathbf{R}^{n}$. Hence we may assume that

$$
C_{1}=\left\|\nabla_{x} J\right\|_{\infty}
$$

in condition (1.10). 
The boundary operator $\Lambda$ is called a first-order, Višik-Ventcel' boundary condition ([39], [41]) and the boundary operator $\gamma_{0} T$ is called a first-order, Lévy-Ventcel' boundary condition ([7]). The boundary operator $L=\Lambda+\gamma_{0} T$ is called a first-order, Ventcel' boundary condition (see [41], [7]). The three terms of $L$

$$
\mu\left(x^{\prime}\right) \frac{\partial u}{\partial \mathbf{n}}, \quad \gamma\left(x^{\prime}\right) u, \quad T u\left(x^{\prime}\right)
$$

are supposed to correspond to the reflection and absorption phenomena at the boundary and the inward jump phenomenon from the boundary, respectively $([32])$. $\Lambda$ :

In this paper we impose the following hypothesis on the boundary condition

$$
\mu\left(x^{\prime}\right)-\gamma\left(x^{\prime}\right)=\mu\left(x^{\prime}\right)+\left|\gamma\left(x^{\prime}\right)\right|>0 \quad \text { on } \partial \Omega .
$$

The intuitive meaning of the hypothesis (1.13) is that either the reflection phenomenon or the absorption phenomenon occurs at every point of the boundary $\partial \Omega$.

It should be emphasized that the boundary condition $\Lambda$ is degenerate from the viewpoint of elliptic boundary value problems. This is due to the fact that the so-called Lopatinski-Shapiro ellipticity condition is violated at each point $x^{\prime}$ of the set $\partial \Omega_{0}=\left\{x^{\prime} \in \partial \Omega: \mu\left(x^{\prime}\right)=0\right\}$ (see [42, Section 11], [33, Section 6.6]). More precisely, the boundary condition $\Lambda$ is non-degenerate (or coercive) if and only if either $\mu\left(x^{\prime}\right)>0$ on $\partial \Omega$ (the regular Robin case) or $\mu\left(x^{\prime}\right) \equiv 0$ and $\gamma\left(x^{\prime}\right)<0$ on $\partial \Omega$ (the Dirichlet case).

We give a simple example of the functions $\mu\left(x^{\prime}\right)$ and $\gamma\left(x^{\prime}\right)$ in condition (1.13) in Euclidean plane $\mathbf{R}^{2}$ :

Example 1.3 Let

$$
\Omega=\left\{\left(x_{1}, x_{2}\right) \in \mathbf{R}^{2}: x_{1}^{2}+x_{2}^{2}<1\right\}
$$

be the unit disk with the boundary

$$
\partial \Omega=\left\{\left(x_{1}, x_{2}\right) \in \mathbf{R}^{2}: x_{1}^{2}+x_{2}^{2}=1\right\} .
$$

For a local coordinate system $x_{1}=\cos \theta, x_{2}=\sin \theta$ with $\theta \in[0,2 \pi]$ on the unit circle $\partial \Omega$, we define two functions $\mu\left(x_{1}, x_{2}\right)$ and $\gamma\left(x_{1}, x_{2}\right)$ as follows:

$$
\mu\left(x_{1}, x_{2}\right)=\mu(\cos \theta, \sin \theta)= \begin{cases}e^{\frac{2}{\pi}-\frac{1}{\theta}\left(1-e^{\frac{2}{\pi}+\frac{1}{\theta-\frac{\pi}{2}}}\right)} & \text { for } \theta \in\left[0, \frac{\pi}{2}\right], \\ 1 & \text { for } \theta \in\left[\frac{\pi}{2}, \pi\right] \\ e^{\frac{2}{\pi}+\frac{1}{\theta-\frac{3 \pi}{2}}}\left(1-e^{\frac{2}{\pi}-\frac{1}{\theta-\pi}}\right) & \text { for } \theta \in\left[\pi, \frac{3 \pi}{2}\right] \\ 0 & \text { for } \theta \in\left[\frac{3 \pi}{2}, 2 \pi\right]\end{cases}
$$

and

$$
\gamma\left(x_{1}, x_{2}\right):=\mu\left(x_{1}, x_{2}\right)-1 \quad \text { on } \partial \Omega
$$


1.1 The case where $T \equiv 0$

The first purpose of this paper is to prove an existence and uniqueness theorem for the non-homogeneous boundary value problem

$$
\begin{cases}W u=(A+S) u=f & \text { in } \Omega, \\ \Lambda u=\mu\left(x^{\prime}\right) \frac{\partial u}{\partial \mathbf{n}}+\gamma\left(x^{\prime}\right) u=\varphi & \text { on } \partial \Omega\end{cases}
$$

under condition (1.13) in the framework of Sobolev spaces $H_{p}^{2+\theta}(\Omega)$ and Besov spaces $B_{p, p}^{1+\theta-1 / p}(\partial \Omega)$ which will be introduced in Section 2 .

The crucial point is how to define a version of Besov spaces in which problem (1.14) is uniquely solvable. In this subsection, following [30] and [33] we introduce a suitable subspace of the classical Besov space $B_{p, p}^{1+\theta}(\partial \Omega)$ which corresponds to our boundary condition $\Lambda$ under condition (1.13).

If condition (1.13) is satisfied, then we can define a modified Besov space corresponding to $\Lambda$ by the formula ([30], [33])

$$
\begin{aligned}
& B_{p, p, \star}^{1+\theta-1 / p}(\partial \Omega):=\mu\left(x^{\prime}\right) B_{p, p}^{1+\theta-1 / p}(\partial \Omega)+\left|\gamma\left(x^{\prime}\right)\right| B_{p, p}^{2+\theta-1 / p}(\partial \Omega) \\
= & \left\{\varphi=\mu\left(x^{\prime}\right) \varphi_{1}-\gamma\left(x^{\prime}\right) \varphi_{2}: \varphi_{1} \in B_{p, p}^{1+\theta-1 / p}(\partial \Omega), \varphi_{2} \in B_{p, p}^{2+\theta-1 / p}(\partial \Omega)\right\} .
\end{aligned}
$$

The space $B_{p, p, \star}^{1+\theta-1 / p}(\partial \Omega)$ is a Banach space with respect to the norm

$$
\begin{aligned}
& \|\varphi\|_{B_{p, p, \star}^{1+\theta-1 / p}(\partial \Omega)} \\
= & \inf \left\{\left\|\varphi_{1}\right\|_{B_{p, p}^{1+\theta-1 / p}(\partial \Omega)}+\left\|\varphi_{2}\right\|_{B_{p, p}^{2+\theta-1 / p}(\partial \Omega)}: \varphi=\mu\left(x^{\prime}\right) \varphi_{1}-\gamma\left(x^{\prime}\right) \varphi_{2}\right\} .
\end{aligned}
$$

The boundary space $B_{p, p, \star}^{1+\theta-1 / p}(\partial \Omega)$ can be understood as an "interpolation space" between the usual Besov spaces $B_{p, p}^{2+\theta-1 / p}(\partial \Omega)$ and $B_{p, p}^{1+\theta-1 / p}(\partial \Omega)$. More precisely, we have the inclusions

$$
B_{p, p}^{2+\theta-1 / p}(\partial \Omega) \subset B_{p, p, \star}^{1+\theta-1 / p}(\partial \Omega) \subset B_{p, p}^{1+\theta-1 / p}(\partial \Omega)
$$

and the relations

$$
\begin{aligned}
& B_{p, p, \star}^{1+\theta-1 / p}(\partial \Omega)=\mu\left(x^{\prime}\right) B_{p, p}^{1+\theta-1 / p}(\partial \Omega)+\left|\gamma\left(x^{\prime}\right)\right| B_{p, p}^{2+\theta-1 / p}(\partial \Omega) \\
= & \begin{cases}B_{p, p}^{2+\theta-1 / p}(\partial \Omega) & \text { if } \mu\left(x^{\prime}\right)=0 \text { on } \partial \Omega \text { (the Dirichlet case), } \\
B_{p, p}^{1+\theta-1 / p}(\partial \Omega) & \text { if } \mu\left(x^{\prime}\right)>0 \text { on } \partial \Omega \text { (the regular Robin case) }\end{cases}
\end{aligned}
$$

under condition (1.13).

Now we are in position to state our existence and uniqueness result for the boundary value problem (1.14):

Theorem 1.1 Let $n<p<\infty$. Assume that conditions (1.4) through (1.6) and condition (1.13) are satisfied. Then the mapping

$$
(W, \Lambda): H_{p}^{2+\theta}(\Omega) \longrightarrow H_{p}^{\theta}(\Omega) \oplus B_{p, p, \star}^{1+\theta-1 / p}(\partial \Omega)
$$


is an algebraic and topological isomorphism for all $0 \leq \theta<\theta_{0}$. In particular, there exists a unique solution $u \in H_{p}^{2+\theta}(\Omega)$ of the non-homogeneous problem (1.14) for any $f \in H_{p}^{\theta}(\Omega)$ and any $\varphi \in B_{p, p, \star}^{1+\theta-1 / p}(\partial \Omega)$.

The proof of Theorem 1.1 will be carried out in several steps. First, in Section 3 we prove some mapping properties of Lévy integro-differential operators defined on $\mathbf{R}^{n}$ (Theorem 3.1) and on $\Omega$ (Theorem 3.2), respectively. In the second part of Theorem 3.2, we derive a compactness result for the Lévy operator $S$. More precisely, we prove that the integral operator

$$
S: H_{p}^{2+\theta}(\Omega) \longrightarrow H_{p}^{\theta}(\Omega)
$$

is compact for all $0 \leq \theta<\theta_{0}$, if $n<p<\infty$. Consequently, the mapping

$$
(W, \Lambda)=(A+S, \Lambda)=(A, \Lambda)+(S, 0)
$$

is a compact perturbation of the operator $(A, \Lambda)$. Thus we are reduced to the study of the case where $S \equiv 0$. However, by virtue of [30, Theorem 1] and [33, Theorem 1.1] we can prove that the mapping

$$
(A, \Lambda): H_{p}^{2+\theta}(\Omega) \longrightarrow H_{p}^{\theta}(\Omega) \oplus B_{p, p, \star}^{1+\theta-1 / p}(\partial \Omega)
$$

is an algebraic and topological isomorphism for all $0 \leq \theta<\theta_{0}$ (Theorem 4.1) in Section 4. Hence, by using a fundamental result of the index theory we obtain that

$$
\text { ind }(W, \Lambda)=\operatorname{ind}(A, \Lambda)=0 \text {. }
$$

Indeed, it is known (see [15, Theorem 2.6], [26, Theorem 5.10]) that the index is stable under compact perturbations. By using the maximum principles (Theorem 6.2 and Lemma 6.1 ) proved in the last Section 6 , we find that $(W, \Lambda)$ is injective (Theorem 4.2). In this way, we can prove that the mapping $(W, \Lambda)$ is bijective. Finally, the continuity of the inverse of the mapping $(W, \Lambda)$ follows immediately from an application of Banach's closed graph theorem ([26, Theorem 3.10], [43, Chapter II, Section 6, Theorem 1]), since the mapping $(W, \Lambda)$ is continuous.

Remark 1.2 Some remarks are in order.

$1^{\circ}$ Problem (1.14) with non-degenerate boundary conditions was studied extensively by Bony-Courrège-Priouret ([7, Théorème XVII $]$ ) in the framework of Hölder spaces $C^{2+\theta}(\bar{\Omega}), 0<\theta<\theta_{0}$. In this case, instead of our hypothesis $H\left(\theta_{0}\right)<\infty$, they consider the following Hölder continuity condition:

$$
A\left(\theta_{0}\right)=\sup _{h, x, z \in \mathbf{R}^{n}}\left(\frac{|K(x+h, z)-K(x, z)|}{|h|^{\theta_{0}}}\right)<\infty .
$$

These results were extended by Bony [5] to the usual Sobolev spaces $W^{2, p}(\Omega)$ for $n<p<\infty$. 
$2^{\circ}$ In the previous works of Taira [30], [31] and [33], we studied problem (1.14) with degenerate boundary conditions under the condition $A\left(\theta_{0}\right)<\infty$. It should be emphasized that $A\left(\theta_{0}\right)<\infty$ implies $H(\theta)<\infty$ for all $0 \leq \theta<\theta_{0}$, while $H\left(\theta_{0}\right)<\infty$ implies $A\left(\theta_{0}\right)<\infty$ (Lemma 2.5) in Section 2.

$3^{\circ}$ Theorem 1.1 is proved by Runst-Youssfi [24, Theorem 1.1], assuming the Hopf boundary point lemma for second-order, elliptic Waldenfels integrodifferential operators with discontinuous coefficients. The present paper is the first time to prove precisely the existence and uniqueness theorem for the boundary value problem (1.14).

1.2 The general case

The second purpose of this paper is to prove an existence and uniqueness theorem for the non-homogeneous Ventcel' boundary value problem

$$
\begin{cases}W u=(A+S) u=f & \text { in } \Omega \\ L u=\left(\Lambda+\gamma_{0} T\right) u=\varphi & \text { on } \partial \Omega\end{cases}
$$

in the framework of Sobolev spaces $H_{p}^{2+\theta}(\Omega)$ and Besov spaces $B_{p, p}^{1+\theta-1 / p}(\partial \Omega)$. Here $\gamma_{0} T$ is a first-order, Lévy-Ventcel' boundary operator defined by formula (1.8). We study the Ventcel' boundary value problem (1.17) under a strong condition (compared with condition (1.13))

$$
\mu\left(x^{\prime}\right)>0 \text { on } \partial \Omega .
$$

It should be emphasized that

$$
B_{p, p, \star}^{1+\theta-1 / p}(\partial \Omega)=B_{p, p}^{1+\theta-1 / p}(\partial \Omega) \text { if } \mu\left(x^{\prime}\right)>0 \text { on } \partial \Omega .
$$

Now we can state our existence and uniqueness result for the Ventcel' boundary value problem (1.17):

Theorem 1.2 Let $0<\theta_{0}<1$ and $n<p<\infty$. Assume that conditions (1.4) through (1.6), conditions (1.9) through (1.12) and condition (1.18) are satisfied. Then the mapping

$$
(W, L): H_{p}^{2+\theta}(\Omega) \longrightarrow H_{p}^{\theta}(\Omega) \oplus B_{p, p}^{1+\theta-1 / p}(\partial \Omega)
$$

is an algebraic and topological isomorphism for all $0 \leq \theta<\min \left\{\theta_{0}, \theta_{1}\right\}$. In particular, there exists a unique solution $u \in H_{p}^{2+\theta}(\Omega)$ of the Ventcel' boundary value problem (1.17) for any $f \in H_{p}^{\theta}(\Omega)$ and any $\varphi \in B_{p, p}^{1+\theta-1 / p}(\partial \Omega)$.

The proof of Theorem 1.2 goes through just as in Section 4 . In fact, in Section 5 we prove that the Lévy-Ventcel' boundary operator

$$
\gamma_{0} T: H_{p}^{2+\theta}(\Omega) \longrightarrow B_{p, p}^{1+\theta-1 / p}(\partial \Omega)
$$


is compact for all $0 \leq \theta<\min \left\{\theta_{0}, \theta_{1}\right\}$ if $n<p<\infty$ (Theorem 5.2). Namely, we find that the mapping

$$
(W, L)=(W, \Lambda)+\left(0, \gamma_{0} T\right): H_{p}^{2+\theta}(\Omega) \longrightarrow H_{p}^{\theta}(\Omega) \oplus B_{p, p}^{1+\theta-1 / p}(\partial \Omega)
$$

is a compact perturbation of the operator $(W, \Lambda)$ under condition (1.18). Therefore, we have the index formula

$$
\text { ind }(W, L)=\operatorname{ind}(W, \Lambda)=0 \text {, }
$$

since the index is stable under compact perturbations. In this way, we are reduced to the study of the mapping $(W, \Lambda)$ (Theorem 1.1). By using the Hopf boundary point lemma (Lemma 6.1). we prove that the mapping $(W, L)$ is injective and hence bijective (Theorem 5.3).

Remark 1.3 Some remarks are in order.

$1^{\circ}$ It should be noticed that Theorem 1.2 is a generalization of Bony-Courrège-Priouret [7, Théorème XVII], Taira [30, Theorem 1] and RunstYoussfi [24, Theorem 1.1] to a class of first-order Ventcel' boundary value problems for elliptic Waldenfels operators.

$2^{\circ}$ The Višik-Ventcel' boundary value problem (1.17) was studied by Anderson [2], [3], Cattiaux [9] and Takanobu-Watanabe [35] from the viewpoint of stochastic analysis (see also Ikeda-Watanabe [16, Chapter IV, Section 7]).

Finally, we give an overview of existence and uniqueness theorems for the problem (1.17) proved by Bony-Courrège-Priouret [7] and by Taira [30] in the framework of Hölder spaces and by Runst-Youssfi [24] in the framework of Sobolev and Besov spaces of $L^{p}$ type (see Table 1.1).

\section{Functions spaces}

In this section, all functions and distributions are defined on Euclidean space $\mathbf{R}^{n}$. As usual, $\mathcal{S}\left(\mathbf{R}^{n}\right)$ denotes the Schwartz space of rapidly decreasing functions and its dual space $\mathcal{S}^{\prime}\left(\mathbf{R}^{n}\right)$ is the space of tempered distributions on $\mathbf{R}^{n}$. For a distribution $f \in \mathcal{S}^{\prime}\left(\mathbf{R}^{n}\right), \widehat{f}$ denotes the Fourier transform of $f$.

In Subsection 2.1 we characterize $L^{p}$ functions by their Littlewood-Paley series (formula (2.3)). In Subsection 2.2 we give Peetre's equivalent definition of Besov spaces and generalized Sobolev spaces (Theorem 2.1). In Subsection 2.3 we characterize generalized Sobolev spaces $H_{p}^{s}$ in terms of Strichartz's norms (Lemma 2.3). Moreover, we prove important relationships between the conditions $H\left(\theta_{0}\right)$ and $A\left(\theta_{0}\right)$ (Lemma 2.5). 


\begin{tabular}{|c|c|c|}
\hline$W=A+S$ & $L=\Lambda+T$ & studied by \\
\hline $\begin{array}{l}K \in C\left(\bar{\Omega} \times \mathbf{R}^{n}\right) \\
\quad A\left(\theta_{0}\right)<\infty\end{array}$ & $\begin{array}{c}\mu>0 \text { on } \partial \Omega \\
T \text { is of order } 1\end{array}$ & $\begin{array}{c}\text { Bony-Courrège-Priouret [7] } \\
\text { (Théorème XVII) }\end{array}$ \\
\hline $\begin{array}{c}K \in C\left(\bar{\Omega} \times \mathbf{R}^{n}\right) \\
\quad A\left(\theta_{0}\right)<\infty\end{array}$ & $\begin{array}{c}\mu-\gamma>0 \text { on } \partial \Omega \\
T \equiv 0\end{array}$ & $\begin{array}{l}\text { Taira [30] } \\
\text { (Theorem 1) }\end{array}$ \\
\hline $\begin{array}{c}K \in L^{\infty}\left(\mathbf{R}^{n} \times \mathbf{R}^{n}\right) \\
H\left(\theta_{0}\right)<\infty\end{array}$ & $\begin{array}{c}\mu-\gamma>0 \text { on } \partial \Omega \\
T \equiv 0\end{array}$ & $\begin{array}{l}\text { the present paper } \\
\text { (Theorem 1.1) }\end{array}$ \\
\hline $\begin{array}{c}K \in L^{\infty}\left(\mathbf{R}^{n} \times \mathbf{R}^{n}\right) \\
H\left(\theta_{0}\right)<\infty\end{array}$ & $\begin{array}{c}\mu>0 \text { on } \partial \Omega \\
T \text { is of order } 1 \\
J \in L^{\infty}\left(\mathbf{R}^{n} \times \mathbf{R}^{n}\right) \\
G\left(\theta_{1}\right)<\infty\end{array}$ & $\begin{array}{l}\text { the present paper } \\
\text { (Theorem 1.2) }\end{array}$ \\
\hline
\end{tabular}

Table 1.1 An overview of existence and uniqueness theorems for problem (1.17)

2.1 The Littlewood-Paley series of tempered distributions

In this subsection we characterize $L^{p}$ functions by their Littlewood-Paley series. We begin with the following elementary lemma (cf. [4, Lemma 6.1.7]):

Lemma 2.1 For a given constant $a>1$, there exists a function $\varphi(\xi) \in$ $C_{0}^{\infty}\left(\mathbf{R}^{n}\right)$ such that

$$
\begin{gathered}
\operatorname{supp} \varphi=\left\{\xi \in \mathbf{R}^{n}: \frac{1}{a} \leq|\xi| \leq a\right\}, \\
\sum_{j \in \mathbf{Z}} \varphi\left(a^{-j} \xi\right)=1 \quad \text { for all } \xi \neq 0 .
\end{gathered}
$$

Furthermore, if we define a function $\psi_{0}(\xi)$ by the formula

$$
\psi_{0}(\xi)=\sum_{j=-\infty}^{0} \varphi\left(a^{-j} \xi\right), \quad \xi \in \mathbf{R}^{n},
$$

then it follows from properties (2.1a) and (2.1b) that

$$
\begin{aligned}
& \psi_{0} \in C_{0}^{\infty}\left(\mathbf{R}^{n}\right) \\
& \operatorname{supp} \psi_{0}=\left\{\xi \in \mathbf{R}^{n}:|\xi| \leq a\right\} \\
& \psi_{0}(\xi)=1-\sum_{j=1}^{\infty} \varphi\left(a^{-j} \xi\right) \text { for all } \xi \neq 0 .
\end{aligned}
$$


Now, by using the Fourier transform we can introduce a family of linear convolution operators

$$
\begin{aligned}
& \Delta_{0}: \mathcal{S}^{\prime}\left(\mathbf{R}^{n}\right) \longrightarrow \mathcal{S}^{\prime}\left(\mathbf{R}^{n}\right), \\
& \Delta_{j}: \mathcal{S}^{\prime}\left(\mathbf{R}^{n}\right) \longrightarrow \mathcal{S}^{\prime}\left(\mathbf{R}^{n}\right), \quad j=1,2, \ldots,
\end{aligned}
$$

by the formulas

$$
\begin{aligned}
& \widehat{\Delta_{0} f}(\xi)=\psi_{0}(\xi) \widehat{f}(\xi), \\
& \widehat{\Delta_{j} f}(\xi)=\varphi\left(a^{-j} \xi\right) \widehat{f}(\xi), \quad j=1,2, \ldots
\end{aligned}
$$

Then, by properties (2.1) and (2.2) it is easy to see that

$$
f=\sum_{j=0}^{\infty} \Delta_{j} f \quad \text { for } f \in \mathcal{S}^{\prime}\left(\mathbf{R}^{n}\right)
$$

Indeed, it suffices to note that, for all $\xi \neq 0$,

$$
\sum_{j=0}^{\infty} \widehat{\Delta_{j} f}(\xi)=\widehat{\Delta_{0} f}(\xi)+\sum_{j=1}^{\infty} \widehat{\Delta_{j} f}(\xi)=\left(\sum_{j \in \mathbf{Z}} \varphi\left(a^{-j} \xi\right)\right) \widehat{f}(\xi)=\widehat{f}(\xi) .
$$

The series (2.3) is called the Littlewood-Paley series of $f$.

\subsection{Peetre's definition of Besov and generalized Sobolev spaces}

In this subsection, following Bergh-Löfström [4] we give Peetre's equivalent definition of Besov spaces and generalized Sobolev spaces.

To do this, we choose $a=2$ in Lemma 2.1. Namely, $\varphi(\xi)$ is a function in $C_{0}^{\infty}\left(\mathbf{R}^{n}\right)$ which satisfies the conditions

$$
\begin{gathered}
\operatorname{supp} \varphi=\left\{\xi \in \mathbf{R}^{n}: \frac{1}{2} \leq|\xi| \leq 2\right\}, \\
\sum_{k \in \mathbf{Z}} \varphi\left(2^{-k} \xi\right)=1 \quad \text { for all } \xi \neq 0 .
\end{gathered}
$$

Then we can define functions $\varphi_{k}(x), \psi(x) \in \mathcal{S}\left(\mathbf{R}^{n}\right)$ by the formulas

$$
\begin{aligned}
& \widehat{\varphi_{k}}(\xi)=\varphi\left(2^{-k} \xi\right), \quad k \in \mathbf{Z}, \\
& \widehat{\psi}(\xi)=\psi_{0}(\xi)=1-\sum_{j=1}^{\infty} \varphi\left(2^{-j} \xi\right) .
\end{aligned}
$$

It should be noticed that

$$
\begin{aligned}
& \operatorname{supp} \widehat{\varphi_{k}}=\left\{\xi \in \mathbf{R}^{n}: 2^{k-1} \leq|\xi| \leq 2^{k+1}\right\}, \quad k \in \mathbf{Z} \\
& \operatorname{supp} \widehat{\psi}=\left\{\xi \in \mathbf{R}^{n}:|\xi| \leq 2\right\}
\end{aligned}
$$


If $s \in \mathbf{R}$, we define the Bessel potential $J^{s}$ by the formula (see [28])

$$
J^{s}=(I-\Delta)^{s / 2}: \mathcal{S}^{\prime}\left(\mathbf{R}^{n}\right) \longrightarrow \mathcal{S}^{\prime}\left(\mathbf{R}^{n}\right) .
$$

More precisely, we let

$$
J^{s} f=\mathcal{F}^{*}\left(\left(1+|\xi|^{2}\right)^{s / 2} \widehat{f}(\xi)\right) \quad \text { for } f \in \mathcal{S}^{\prime}\left(\mathbf{R}^{n}\right) .
$$

Then we have the following basic properties of the objects just defined (see [4, Lemma 6.2.1]):

Lemma 2.2 (i) Assume that a distribution $f \in \mathcal{S}^{\prime}\left(\mathbf{R}^{n}\right)$ satisfies the condition

$$
\varphi_{k} * f \in L^{p}\left(\mathbf{R}^{n}\right), \quad k \in \mathbf{Z},
$$

with $1 \leq p \leq \infty$. Then we have, for all $s \in \mathbf{R}$,

$$
\left\|J^{s}\left(\varphi_{k} * f\right)\right\|_{L^{p}\left(\mathbf{R}^{n}\right)} \leq C 2^{s k}\left\|\varphi_{k} * f\right\|_{L^{p}\left(\mathbf{R}^{n}\right)}, \quad k=1,2, \ldots,
$$

with a constant $C>0$ independent of $p$ and $k$.

(ii) If a distribution $f \in \mathcal{S}^{\prime}\left(\mathbf{R}^{n}\right)$ satisfies the condition

$$
\psi * f \in L^{p}\left(\mathbf{R}^{n}\right),
$$

with $1 \leq p \leq \infty$, then we have, for all $s \in \mathbf{R}$,

$$
\left\|J^{s}(\psi * f)\right\|_{L^{p}\left(\mathbf{R}^{n}\right)} \leq C^{\prime}\|\psi * f\|_{L^{p}\left(\mathbf{R}^{n}\right)},
$$

with a constant $C^{\prime}>0$ independent of $p$ and $k$.

Now, by virtue of Lemma 2.2 we can make the following definition 2.1 of the Besov and generalized Sobolev spaces (see [4, Definition 6.2.2]):

Definition 2.1 Let $s \in \mathbf{R}$, and $1 \leq p, q \leq \infty$. If $f \in \mathcal{S}^{\prime}\left(\mathbf{R}^{n}\right)$, we let

$$
\begin{aligned}
& \|f\|_{B_{p, q}^{s}\left(\mathbf{R}^{n}\right)} \\
= & \begin{cases}\|\psi * f\|_{L^{p}\left(\mathbf{R}^{n}\right)}+\left(\sum_{k=1}^{\infty}\left(2^{s k}\left\|\varphi_{k} * f\right\|_{L^{p}\left(\mathbf{R}^{n}\right)}\right)^{q}\right)^{1 / q} & \text { if } 1 \leq q<\infty, \\
\|\psi * f\|_{L^{p}\left(\mathbf{R}^{n}\right)}+\sup _{k \geq 1}\left(2^{s k}\left\|\varphi_{k} * f\right\|_{L^{p}\left(\mathbf{R}^{n}\right)}\right) & \text { if } q=\infty,\end{cases}
\end{aligned}
$$

and

$$
\|f\|_{H_{p}^{s}\left(\mathbf{R}^{n}\right)}=\left\|J^{s} f\right\|_{L^{p}\left(\mathbf{R}^{n}\right)} .
$$

Then the Besov space $B_{p, q}^{s}\left(\mathbf{R}^{n}\right)$ and the generalized Sobolev space $H_{p}^{s}\left(\mathbf{R}^{n}\right)$ are defined respectively as follows:

$$
\begin{aligned}
& B_{p, q}^{s}\left(\mathbf{R}^{n}\right)=\left\{f \in \mathcal{S}^{\prime}\left(\mathbf{R}^{n}\right):\|f\|_{B_{p, q}^{s}\left(\mathbf{R}^{n}\right)}<\infty\right\}, \\
& H_{p}^{s}\left(\mathbf{R}^{n}\right)=\left\{f \in \mathcal{S}^{\prime}\left(\mathbf{R}^{n}\right):\|f\|_{H_{p}^{s}\left(\mathbf{R}^{n}\right)}<\infty\right\} .
\end{aligned}
$$


Remark 2.1 It is known (see Bergh-Löfström [4], Triebel [36, Chapter 2]) that the Sobolev space $H^{s, p}\left(\mathbf{R}^{n}\right)$ and the usual Besov space $B^{s, p}\left(\mathbf{R}^{n-1}\right)$ are respectively equivalent to the following:

$$
\begin{aligned}
& H^{s, p}\left(\mathbf{R}^{n}\right)=H_{p}^{s}\left(\mathbf{R}^{n}\right) \quad \text { for all } s \in \mathbf{R} \text { and } 1<p<\infty, \\
& B^{s, p}\left(\mathbf{R}^{n-1}\right)=B_{p, p}^{s}\left(\mathbf{R}^{n-1}\right) \quad \text { for all } s \in \mathbf{R} \text { and } 1 \leq p \leq \infty .
\end{aligned}
$$

It should be noticed that

$$
H_{p}^{0}\left(\mathbf{R}^{n}\right)=L^{p}\left(\mathbf{R}^{n}\right) \quad \text { for all } 1 \leq p \leq \infty
$$

Furthermore, it is easy to verify the following two assertions (I) and (II) ([36, Chapter 2]):

(I) The function spaces $B_{p, q}^{s}\left(\mathbf{R}^{n}\right)$ and $H_{p}^{s}\left(\mathbf{R}^{n}\right)$ are Banach spaces with the norms $\|\cdot\|_{B_{p, q}^{s}\left(\mathbf{R}^{n}\right)}$ and $\|\cdot\|_{H_{p}^{s}\left(\mathbf{R}^{n}\right)}$, respectively.

(II) The Bessel potential $J^{\sigma}$ maps $B_{p, q}^{s}\left(\mathbf{R}^{n}\right)$ isomorphically onto $B_{p, q}^{s-\sigma}\left(\mathbf{R}^{n}\right)$ for each $\sigma \in \mathbf{R}$, and it maps $H_{p}^{s}\left(\mathbf{R}^{n}\right)$ isomorphically onto $H_{p}^{s-\sigma}\left(\mathbf{R}^{n}\right)$ for each $\sigma \in \mathbf{R}$, respectively.

The next theorem characterizes the spaces $B_{p, q}^{s}\left(\mathbf{R}^{n}\right)$ and $H_{p}^{s}\left(\mathbf{R}^{n}\right)$ in terms of the Littlewood-Paley series (see [4, Theorem 6.4.3]):

Theorem 2.1 We have the following characterizations of the spaces $B_{p, q}^{s}\left(\mathbf{R}^{n}\right)$ and $H_{p}^{s}\left(\mathbf{R}^{n}\right)$ :

(i) Let $s \in \mathbf{R}$ and $1<p<\infty$. Then we have, for any $f \in \mathcal{S}^{\prime}\left(\mathbf{R}^{n}\right)$,

$$
f \in H_{p}^{s}\left(\mathbf{R}^{n}\right) \Longleftrightarrow\left(\sum_{j=0}^{\infty} 2^{2 s j}\left|\Delta_{j} f\right|^{2}\right)^{1 / 2} \in L^{p}\left(\mathbf{R}^{n}\right) .
$$

(ii) Let $s \in \mathbf{R}$ and $1 \leq p, q \leq \infty$. Then we have, for any $f \in \mathcal{S}^{\prime}\left(\mathbf{R}^{n}\right)$,

$$
f \in B_{p, q}^{s}\left(\mathbf{R}^{n}\right) \Longleftrightarrow \sum_{j=0}^{\infty} 2^{s q j}\left\|\Delta_{j} f\right\|_{B_{p, q}^{s}\left(\mathbf{R}^{n}\right)}<\infty .
$$

We remark that

$$
H_{p}^{s}\left(\mathbf{R}^{n}\right) \subset B_{p, \infty}^{s}\left(\mathbf{R}^{n}\right)
$$

and that

$$
B_{p, \infty}^{s^{\prime}}\left(\mathbf{R}^{n}\right) \subset H_{p}^{s}\left(\mathbf{R}^{n}\right) \text { if } s<s^{\prime} .
$$

Now let $\Omega$ be a bounded domain in $\mathbf{R}^{n}$ with smooth boundary $\partial \Omega$. In order to define function spaces on $\Omega$, we make use of restriction arguments (see [36, Chapter 3])

Let $\mathcal{D}^{\prime}(\Omega)$ be the space of distributions on $\Omega$. Then we let

$$
H_{p}^{s}(\Omega)=\left\{f \in \mathcal{D}^{\prime}(\Omega): f=\left.g\right|_{\Omega} \text { for some } g \in H_{p}^{s}\left(\mathbf{R}^{n}\right)\right\}
$$


with the norm

$$
\|f\|_{H_{p}^{s}(\Omega)}=\inf \left\{\|g\|_{H_{p}^{s}\left(\mathbf{R}^{n}\right)}:\left.g\right|_{\Omega}=f \text { for } g \in H_{p}^{s}\left(\mathbf{R}^{n}\right)\right\} .
$$

Similarly, we define the Besov space

$$
B_{p, q}^{s}(\Omega)=\left\{f \in \mathcal{D}^{\prime}(\Omega): f=\left.g\right|_{\Omega} \text { for some } g \in B_{p, q}^{s}\left(\mathbf{R}^{n}\right)\right\}
$$

with the norm

$$
\|f\|_{B_{p, q}^{s}(\Omega)}=\inf \left\{\|g\|_{B_{p, q}^{s}\left(\mathbf{R}^{n}\right)}:\left.g\right|_{\Omega}=f \text { for } g \in B_{p, q}^{s}\left(\mathbf{R}^{n}\right)\right\}
$$

(see, for example, [37] and [23]).

2.3 Generalized Sobolev spaces and Strichartz's norms

For the proof of Theorem 1.1, we make use of the following characterization of generalized Sobolev spaces $H_{p}^{s}$ in terms of Strichartz's norms, which may be found, for example in [37, p. 194, Theorem 3.5.3].

Lemma 2.3 Let $0<s<1$ and $1<p<\infty$. Then the quantity

$$
N_{p}^{s}(g)=\|g\|_{L^{p}\left(\mathbf{R}^{n}\right)}+\left\|L_{s}(g)\right\|_{L^{p}\left(\mathbf{R}^{n}\right)}
$$

defines an equivalent norm on the Sobolev space $H_{p}^{s}\left(\mathbf{R}^{n}\right)$, where

$$
\begin{aligned}
& L_{s}(g)(x) \\
& = \begin{cases}\left(\int_{\mathbf{R}^{n}}|g(x+h)-g(x)|^{2} \frac{d h}{|h|^{n+2 s}}\right)^{1 / 2} & \text { if } p \geq 2 \\
\left(\int_{0}^{\infty}\left(\int_{\{0<|h| \leq 1\}}|g(x+t h)-g(x)| d h\right)^{2} \frac{d t}{t^{1+2 s}}\right)^{1 / 2} & \text { if } 1<p<2\end{cases}
\end{aligned}
$$

Moreover, we need the following lemma:

Lemma 2.4 Let $1<p<\infty$ and $0 \leq \gamma \leq 1$. If we let

$$
T_{h}(f)(x):=f(x+h)-f(x),
$$

then there exists a constant $C>0$, depending on $\gamma$, such that

$$
\left\|T_{h}(f)\right\|_{H_{p}^{\gamma}\left(\mathbf{R}^{n}\right)} \leq C|h|\|f\|_{H_{p}^{1+\gamma}\left(\mathbf{R}^{n}\right)}
$$

for all $f \in H^{1+\gamma, p}\left(\mathbf{R}^{n}\right)$ (see Figure 2.1). 




Fig. 2.1 The mapping properties of $T_{h}$ for $1<p<\infty$ and $0 \leq \gamma \leq 1$ in Lemma 2.4

Proof The proof of Lemma 2.4 is divided into three steps.

Step (1): The case where $\gamma=0$ (cf. Ziemer [44, Theorem 2.1.6]). We have only to show that

$$
\|f(\cdot+h)-f(\cdot)\|_{L^{p}\left(\mathbf{R}^{n}\right)} \leq|h|\|\nabla f\|_{L^{p}\left(\mathbf{R}^{n}\right)} \quad \text { for all } f \in C_{0}^{\infty}\left(\mathbf{R}^{n}\right),
$$

since the space $C_{0}^{\infty}\left(\mathbf{R}^{n}\right)$ is dense in $H_{p}^{1}\left(\mathbf{R}^{n}\right)=W^{1, p}\left(\mathbf{R}^{n}\right)$.

To do so, we make use of the formula for $h \neq 0$

$$
\begin{aligned}
\frac{f(x+h)-f(x)}{|h|} & =\frac{1}{|h|} \int_{0}^{|h|} \frac{d}{d t}\left(f\left(x+t \frac{h}{|h|}\right)\right) d t \\
& =\frac{1}{|h|} \int_{0}^{|h|} \nabla f\left(x+t \frac{h}{|h|}\right) \cdot \frac{h}{|h|} d t .
\end{aligned}
$$

Therefore, by applying Minkowskii's inequality for integrals (see [11, p. 194, Theorem 6.19]) to formula (2.8) we obtain that

$$
\begin{aligned}
& \frac{\|f(\cdot+h)-f(\cdot)\|_{L^{p}\left(\mathbf{R}^{n}\right)}}{|h|} \\
\leq & \frac{1}{|h|} \int_{0}^{|h|}\left\|\nabla f\left(\cdot+t \frac{h}{|h|}\right)\right\|_{L^{p}\left(\mathbf{R}^{n}\right)} d t=\frac{1}{|h|} \int_{0}^{|h|}\|\nabla f\|_{L^{p}\left(\mathbf{R}^{n}\right)} d t \\
= & \|\nabla f\|_{L^{p}\left(\mathbf{R}^{n}\right)} .
\end{aligned}
$$

This proves the desired inequality (2.7).

Step (2): The case where $\gamma=1$. We remark that

$$
\begin{aligned}
\left\|T_{h}(f)\right\|_{H_{p}^{1}\left(\mathbf{R}^{n}\right)} & \leq C\left(\sum_{j=1}^{n}\left\|\frac{\partial T_{h}(f)}{\partial x_{j}}\right\|_{L^{p}\left(\mathbf{R}^{n}\right)}+\left\|T_{h}(f)\right\|_{L^{p}\left(\mathbf{R}^{n}\right)}\right) \\
& \leq C\left(\sum_{j=1}^{n}\left\|T_{h}\left(\frac{\partial f}{\partial x_{j}}\right)\right\|_{L^{p}\left(\mathbf{R}^{n}\right)}+\left\|T_{h}(f)\right\|_{L^{p}\left(\mathbf{R}^{n}\right)}\right) .
\end{aligned}
$$


However, it follows from Step (1) that

$$
\begin{aligned}
& \left\|T_{h}\left(\frac{\partial f}{\partial x_{j}}\right)\right\|_{L^{p}\left(\mathbf{R}^{n}\right)}+\left\|T_{h}(f)\right\|_{L^{p}\left(\mathbf{R}^{n}\right)} \\
\leq & C|h|\left(\left\|\frac{\partial f}{\partial x_{j}}\right\|_{H_{p}^{1}\left(\mathbf{R}^{n}\right)}+\|f\|_{L^{p}\left(\mathbf{R}^{n}\right)}\right) .
\end{aligned}
$$

Therefore, by combining inequalities (2.9) and (2.10) we obtain the desired inequality for $\gamma=1$

$$
\left\|T_{h}(f)\right\|_{H_{p}^{1}\left(\mathbf{R}^{n}\right)} \leq C|h|\|f\|_{H_{p}^{2}\left(\mathbf{R}^{n}\right)} .
$$

Step (3): The case where $0<\gamma<1$. From Steps (1) and (2), we obtain that the operator $T_{h}$ is bounded on $H_{p}^{1}\left(\mathbf{R}^{n}\right)=W^{1, p}\left(\mathbf{R}^{n}\right)$ into $L^{p}\left(\mathbf{R}^{n}\right)$ and on $H_{p}^{2}\left(\mathbf{R}^{n}\right)=W^{2, p}\left(\mathbf{R}^{n}\right)$ into $H_{p}^{1}\left(\mathbf{R}^{n}\right)$, respectively. Then, by using an interpolation argument of linear operators we obtain that

$$
T_{h}: H_{p}^{1+\gamma}\left(\mathbf{R}^{n}\right) \longrightarrow H_{p}^{\gamma}\left(\mathbf{R}^{n}\right)
$$

is bounded for all $0<\gamma<1$, with the inequality

$$
\left\|T_{h}(f)\right\|_{H_{p}^{\gamma}\left(\mathbf{R}^{n}\right)} \leq C|h|\|f\|_{H_{p}^{1+\gamma}\left(\mathbf{R}^{n}\right)} \quad \text { for all } f \in H_{p}^{1+\gamma}\left(\mathbf{R}^{n}\right) .
$$

The proof of Lemma 2.4 is complete.

The next lemma describes important relationships between the conditions $H\left(\theta_{0}\right)$ and $A\left(\theta_{0}\right)$ (see [36], [23]):

Lemma 2.5 Let $0<\theta_{0}<1$. Then we have the following relationships between conditions $H\left(\theta_{0}\right)$ and $A\left(\theta_{0}\right)$ :

(i) There exists a constant $C_{1}\left(\theta_{0}\right)>0$ such that

$$
\begin{aligned}
& \sup _{x, h \in \mathbf{R}^{n}}\left(\frac{|g(x+h)-g(x)|}{|h|^{\theta_{0}}}\right) \\
& \leq C_{1}\left(\theta_{0}\right)\left(\sup _{x \in \mathbf{R}^{n}}\left(\int_{\mathbf{R}^{n}}|g(x+h)-g(x)|^{2} \frac{d h}{|h|^{n+2 \theta_{0}}}\right)^{1 / 2}+\|g\|_{\infty}\right) \\
& \quad \text { for all } g \in L^{\infty}\left(\mathbf{R}^{n}\right) .
\end{aligned}
$$

(ii) Conversely, for every $0 \leq \theta<\theta_{0}<1$ there exists a constant $C_{2}(\theta)>0$ such that

$$
\begin{aligned}
& \sup _{x \in \mathbf{R}^{n}}\left(\int_{\mathbf{R}^{n}}|g(x+h)-g(x)|^{2} \frac{d h}{|h|^{n+2 \theta}}\right)^{1 / 2} \\
& \leq C_{2}(\theta)\left(\sup _{x, h \in \mathbf{R}^{n}} \frac{|g(x+h)-g(x)|}{|h|^{\theta_{0}}}+\|g\|_{\infty}\right) \quad \text { for all } g \in L^{\infty}\left(\mathbf{R}^{n}\right) .
\end{aligned}
$$

(iii) If $K \in L^{\infty}\left(\mathbf{R}^{n} \times \mathbf{R}^{n}\right)$, then we have the following two assertions: 
(a) $H\left(\theta_{0}\right)<\infty$ implies that $A\left(\theta_{0}\right)<\infty$.

(b) $A\left(\theta_{0}\right)<\infty$ implies that $H(\theta)<\infty$ for all $0 \leq \theta<\theta_{0}$.

Proof Indeed, we have, for $0<s<1$,

$$
\|g\|_{B_{\infty, \infty}^{s}}=\|g\|_{\infty}+\sup _{x, h \in \mathbf{R}^{n}} \frac{|g(x+h)-g(x)|}{|h|^{s}}
$$

and

$$
\|g\|_{B_{\infty, 2}^{s}}=\|g\|_{\infty}+\sup _{x \in \mathbf{R}^{n}}\left(\int_{\mathbf{R}^{n}}|g(x+h)-g(x)|^{2} \frac{d h}{|h|^{n+2 s}}\right)^{1 / 2} .
$$

Therefore, the lemma follows from the embeddings (see [36, Section 2.7])

$$
B_{\infty, 2}^{\theta_{0}}\left(\mathbf{R}^{n}\right) \subset B_{\infty, \infty}^{\theta_{0}}\left(\mathbf{R}^{n}\right) \subset B_{\infty, 2}^{\theta}\left(\mathbf{R}^{n}\right) \quad \text { for } 0 \leq \theta<\theta_{0} .
$$

The proof of Lemma 2.5 is complete.

\section{Mapping properties of Lévy operators}

In this section we prove some mapping properties of Lévy integro-differential operators defined on $\mathbf{R}^{n}$ (Theorem 3.1) in Subsection 3.1 and on $\Omega$ (Theorem 3.2 ) in Subsection 3.2, respectively.

\subsection{Boundedness of Lévy operators defined on $\mathbf{R}^{n}$}

In this subsection, we consider the Lévy integro-differential operator

$$
S u(x)=\int_{\mathbf{R}^{n} \backslash\{0\}}\left(u(x+z)-u(x)-\sum_{j=1}^{n} z_{j} \frac{\partial u}{\partial x_{j}}(x)\right) K(x, z) \mu(d z),
$$

and prove that the Lévy operator

$$
S: H_{p}^{\theta+2}\left(\mathbf{R}^{n}\right) \longrightarrow H_{p}^{\theta}\left(\mathbf{R}^{n}\right)
$$

is bounded for all $1<p<\infty$.

Our main result in this section is stated as follows:

Theorem 3.1 Let $1<p<\infty$ and $0<\theta_{0}<1$. Assume that the growth condition (1.4) and the moment condition (1.6) are satisfied. Then the Lévy operator

$$
S: H_{p}^{2+\theta}\left(\mathbf{R}^{n}\right) \longrightarrow H_{p}^{\theta}\left(\mathbf{R}^{n}\right)
$$

is bounded for all $0 \leq \theta \leq \theta_{0}$. Namely, there exists a constant $C>0$, depending on $p$ and $\theta$, such that

$$
\|S u\|_{H_{p}^{\theta}\left(\mathbf{R}^{n}\right)} \leq C\|u\|_{H_{p}^{2+\theta}\left(\mathbf{R}^{n}\right)} \quad \text { for all } u \in H_{p}^{2+\theta}\left(\mathbf{R}^{n}\right)
$$


Remark 3.1 By part (iii) of Lemma 2.5, we find that $H(\theta)<\infty$ for all $0 \leq$ $\theta \leq \theta_{0}$.

Theorem 3.1 is proved by Taira [30, Lemmas 1.6 and 1.7] in the framework of Hölder spaces under the condition $A\left(\theta_{0}\right)<\infty$. In this paper we give a simpler proof by using real analysis techniques.

First, we shall show that the mapping $S$ can be considered as a bounded operator from $H^{2, p}\left(\mathbf{R}^{n}\right)$ to $L^{p}\left(\mathbf{R}^{n}\right)$ (the case $\theta=0$ ). More precisely, we shall prove the inequality

$$
\begin{aligned}
& \|S u\|_{L^{p}\left(\mathbf{R}^{n}\right)} \\
\leq & C\|K\|_{\infty}\left(\int_{\{|z|>1\}}|z| \mu(d z)+\int_{\{0<|z| \leq 1\}}|z|^{2} \mu(d z)\right)\|u\|_{H_{p}^{2}\left(\mathbf{R}^{n}\right)}
\end{aligned}
$$

for all $u \in H_{p}^{2}\left(\mathbf{R}^{n}\right)$.

Secondly, we shall show the inequality

$$
\begin{aligned}
& \left\|L_{\theta}(S u)\right\|_{L^{p}\left(\mathbf{R}^{n}\right)} \\
\leq & C\left(\|K\|_{\infty}+H(\theta)\right)\left(\int_{\{|z|>1\}}|z| \mu(d z)+\int_{\{0<|z| \leq 1\}}|z|^{2} \mu(d z)\right)\|u\|_{H_{p}^{\theta+2}\left(\mathbf{R}^{n}\right)}
\end{aligned}
$$

for all $u \in H_{p}^{2+\theta}\left(\mathbf{R}^{n}\right)$,

where

$$
L_{\theta}(S u)(x)=\left(\int_{\mathbf{R}^{n}} \frac{|S u(x+h)-S u(x)|^{2}}{|h|^{n+2 \theta}} d h\right)^{1 / 2}, \quad 0<\theta \leq \theta_{0} .
$$

By virtue of Lemma 2.3, the desired inequality (3.1) follows from inequalities (3.2) and (3.3).

\subsubsection{The estimate of $\|S u\|_{L^{p}\left(\mathbf{R}^{n}\right)}$ for $\theta=0$}

In order to prove the $L^{p}$-boundedness, we write $S u(x)$ in the form

$$
S u(x)=S_{1} u(x)+S_{2} u(x) .
$$

Here:

$$
\begin{aligned}
& S_{1} u(x):=\int_{\{|z|>1\}}\left(u(x+z)-u(x)-\sum_{j=1}^{n} z_{j} \frac{\partial u}{\partial x_{j}}(x)\right) K(x, z) \mu(d z), \\
& S_{2} u(x):=\int_{\{0<|z| \leq 1\}}\left(u(x+z)-u(x)-\sum_{j=1}^{n} z_{j} \frac{\partial u}{\partial x_{j}}(x)\right) K(x, z) \mu(d z) .
\end{aligned}
$$

(1) First, we estimate the norm $\left\|S_{1} u\right\|_{L^{p}\left(\mathbf{R}^{n}\right)}$ : If we let

$$
g_{1}(x):=\int_{\{|z|>1\}}|u(x+z)-u(x)| \mu(d z)
$$


and

$$
g_{2}(x):=\sum_{j=1}^{n}\left|\frac{\partial u}{\partial x_{j}}(x)\right| \int_{\{|z|>1\}}\left|z_{j}\right| \mu(d z),
$$

then we have the inequality

$$
\left|S_{1} u(x)\right| \leq\|K\|_{\infty}\left(g_{1}(x)+g_{2}(x)\right) .
$$

However, it follows from an application of Minkowskii's inequality for integrals (see $[11$, p. 194, Theorem 6.19]) and inequality (2.6) with $\gamma:=0$ that

$$
\begin{aligned}
\left\|g_{1}\right\|_{L^{p}\left(\mathbf{R}^{n}\right)} & \leq \int_{\{|z|>1\}}\left(\int_{\mathbf{R}^{n}}|u(x+z)-u(x)|^{p} d x\right)^{1 / p} \mu(d z) \\
& \leq C\left(\int_{\{|z|>1\}}|z| \mu(d z)\right)\|u\|_{H_{p}^{1}\left(\mathbf{R}^{n}\right)} .
\end{aligned}
$$

Moreover, we have the inequality

$$
\begin{aligned}
\left\|g_{2}\right\|_{L^{p}\left(\mathbf{R}^{n}\right)} & \leq \sum_{j=1}^{n}\left\|\frac{\partial u}{\partial x_{j}}\right\|_{L^{p}\left(\mathbf{R}^{n}\right)}\left(\sum_{j=1}^{n} \int_{\{|z|>1\}}\left|z_{j}\right| \mu(d z)\right) \\
& \leq C\left(\int_{\{|z|>1\}}|z| \mu(d z)\right)\|u\|_{H_{p}^{1}\left(\mathbf{R}^{n}\right)} .
\end{aligned}
$$

By combining inequalities (3.4), (3.5) and (3.6), we obtain that

$$
\begin{aligned}
\left\|S_{1} u\right\|_{L^{p}\left(\mathbf{R}^{n}\right)} & \leq\|K\|_{\infty}\left(\left\|g_{1}\right\|_{L^{p}\left(\mathbf{R}^{n}\right)}+\left\|g_{2}\right\|_{L^{p}\left(\mathbf{R}^{n}\right)}\right) \\
& \leq C\|K\|_{\infty}\left(\int_{\{|z|>1\}}|z| \mu(d z)\right)\|u\|_{H_{p}^{1}\left(\mathbf{R}^{n}\right)} .
\end{aligned}
$$

(2) In order to estimate the norm $\left\|S_{2} u\right\|_{L^{p}\left(\mathbf{R}^{n}\right)}$, by using Taylor's formula we obtain that

$$
u(x+z)-u(x)-\sum_{k=1}^{n} z_{k} \frac{\partial u}{\partial x_{k}}(x)=\sum_{k=1}^{n} z_{k} \int_{0}^{1}\left(\frac{\partial u}{\partial x_{k}}(x+t z)-\frac{\partial u}{\partial x_{k}}(x)\right) d t .
$$

Hence we have the inequality

$$
\left|S_{2} u(x)\right| \leq\|K\|_{\infty} \sum_{k=1}^{n} \int_{0}^{1} \int_{\{0<|z| \leq 1\}}|z|\left|\frac{\partial u}{\partial x_{k}}(x+t z)-\frac{\partial u}{\partial x_{k}}(x)\right| \mu(d z) d t .
$$

By Minkowskii's inequality for integrals, it follows that

$$
\begin{aligned}
& \left\|S_{2} u\right\|_{L^{p}\left(\mathbf{R}^{n}\right)} \\
\leq & C\|K\|_{\infty} \\
& \times \int_{0}^{1} \int_{\{0<|z| \leq 1\}}|z| \sum_{k=1}^{n}\left(\int_{\mathbf{R}^{n}}\left|\frac{\partial u}{\partial x_{k}}(x+t z)-\frac{\partial u}{\partial x_{k}}(x)\right|^{p} d x\right)^{1 / p} \mu(d z) d t .
\end{aligned}
$$


However, by applying inequality (2.6) with $\gamma:=0$ we obtain tha6

$$
\begin{aligned}
& \sum_{k=1}^{n}\left(\int_{\mathbf{R}^{n}}\left|\frac{\partial u}{\partial x_{k}}(x+t z)-\frac{\partial u}{\partial x_{k}}(x)\right|^{p} d x\right)^{1 / p} \leq C t|z| \sum_{k=1}^{n}\left\|\frac{\partial u}{\partial x_{k}}\right\|_{H_{p}^{1}\left(\mathbf{R}^{n}\right)} \\
\leq & C t|z|\|u\|_{H_{p}^{2}\left(\mathbf{R}^{n}\right)} \quad \text { for all } 0 \leq \theta \leq 1 .
\end{aligned}
$$

Therefore, we have, by inequality (3.8),

$$
\begin{aligned}
\left\|S_{2} u\right\|_{L^{p}\left(\mathbf{R}^{n}\right)} & \leq C\|K\|_{\infty}\|u\|_{H_{p}^{2}\left(\mathbf{R}^{n}\right)} \int_{0}^{1} \int_{\{0<|z| \leq 1\}} t|z|^{2} \mu(d z) d t \\
& \leq C\|K\|_{\infty}\left(\int_{\{0<|z| \leq 1\}}|z|^{2} \mu(d z)\right)\|u\|_{H_{p}^{2}\left(\mathbf{R}^{n}\right) .}
\end{aligned}
$$

The desired inequality (3.2) follows by combining inequalities (3.7) and (3.9)

$$
\begin{aligned}
\|S u\|_{L^{p}\left(\mathbf{R}^{n}\right)} & \leq\left\|S_{1} u\right\|_{L^{p}\left(\mathbf{R}^{n}\right)}+\left\|S_{2} u\right\|_{L^{p}\left(\mathbf{R}^{n}\right)} \\
& \leq C\|K\|_{\infty}\left(\int_{\{|z|>1\}}|z| \mu(d z)+\int_{\{0<|z| \leq 1\}}|z|^{2} \mu(d z)\right)\|u\|_{H_{p}^{2}\left(\mathbf{R}^{n}\right)}
\end{aligned}
$$

3.1.2 The estimate of $\left\|L_{\theta}(S u)\right\|_{L^{p}\left(\mathbf{R}^{n}\right)}$ for $0<\theta \leq \theta_{0}$

In the following, it suffices to consider the case where $p \geq 2$. In fact, the case where $1<p \leq 2$ can be handled by duality as follows:

$$
p=\frac{q}{q-1}, \quad 2 \leq q<\infty .
$$

(A): The estimate of $L_{\theta}\left(S_{1} u\right)$ : First, we prove the inequality

$$
\left\|L_{\theta}\left(S_{1} u\right)\right\|_{L^{p}\left(\mathbf{R}^{n}\right)} \leq C\left(\|K\|_{\infty}+H(\theta)\right) \int_{\{|z|>1\}}|z| \mu(d z) \cdot\|u\|_{H_{p}^{\theta+1}\left(\mathbf{R}^{n}\right)} .
$$

To do this, we write the difference

$$
S_{1} u(x+h)-S_{1} u(x)
$$

in the following form:

$$
\begin{aligned}
& S_{1} u(x+h)-S_{1} u(x) \\
= & \int_{\{|z|>1\}}(u(x+h+z)-u(x+z)+u(x)-u(x+h)) K(x+h, z) \mu(d z) \\
& +\int_{\{|z|>1\}}(u(x+z)-u(x))(K(x+h, z)-K(x, z)) \mu(d z) \\
& +\sum_{k=1}^{n}\left(\frac{\partial u}{\partial x_{k}}(x)-\frac{\partial u}{\partial x_{k}}(x+h)\right) \int_{\{|z|>1\}} z_{k} K(x+h, z) \mu(d z)
\end{aligned}
$$




$$
\begin{aligned}
& \left.+\sum_{k=1}^{n} \frac{\partial u}{\partial x_{k}}(x) \int_{\{|z|>1\}} z_{k}(K(x, z)-K(x+h, z)) d t\right) \mu(d z) \\
:= & A_{1}(x, h)+A_{2}(x, h)+A_{3}(x, h)+A_{4}(x, h) .
\end{aligned}
$$

Then we have the inequality

$$
\begin{aligned}
L_{\theta}\left(S_{1} u\right)(x)= & \left(\int_{\mathbf{R}^{n}} \frac{\left|S_{1} u(x+h)-S_{2} u(x)\right|^{2}}{|h|^{n+2 \theta}} d h\right)^{1 / 2} \\
\leq & C\left(\int_{\mathbf{R}^{n}} \frac{\left|A_{1}(x, h)\right|^{2}}{|h|^{n+2 \theta}} d h+\int_{\mathbf{R}^{n}} \frac{\left|A_{2}(x, h)\right|^{2}}{|h|^{n+2 \theta}} d h\right. \\
& \left.+\int_{\mathbf{R}^{n}} \frac{\left|A_{3}(x, h)\right|^{2}}{|h|^{n+2 \theta}} d h+\int_{\mathbf{R}^{n}} \frac{\left|A_{4}(x, h)\right|^{2}}{|h|^{n+2 \theta}} d h\right)^{1 / 2} \\
\leq & C\left(\int_{\mathbf{R}^{n}} \frac{\left|A_{1}(x, h)\right|^{2}}{|h|^{n+2 \theta}} d h\right)^{1 / 2}+\left(\int_{\mathbf{R}^{n}} \frac{\left|A_{2}(x, h)\right|^{2}}{|h|^{n+2 \theta}} d h\right)^{1 / 2} \\
& \left.+\left(\int_{\mathbf{R}^{n}} \frac{\left|A_{3}(x, h)\right|^{2}}{|h|^{n+2 \theta}} d h\right)^{1 / 2}+\left(\int_{\mathbf{R}^{n}} \frac{\left|A_{4}(x, h)\right|^{2}}{|h|^{n+2 \theta}} d h\right)^{1 / 2}\right) .
\end{aligned}
$$

(1) The estimate of $A_{1}(x, h)$ : We have, by Taylor's formula,

$$
\begin{aligned}
& A_{1}(x, h) \\
= & \left(\int_{\{|z|>1\}}^{1} \sum_{0=1}^{n}\left(\frac{\partial u}{\partial x_{k}}(x+h+t z)-\frac{\partial u}{\partial x_{k}}(x+t z)\right) z_{k} d t\right) K(x+h, z) \mu(d z) .
\end{aligned}
$$

Since $p \geq 2$, it follows from definition (2.1) and Minkowskii's inequality for integrals that

$$
\begin{aligned}
& \left(\int_{\mathbf{R}^{n}}\left(\int_{\mathbf{R}^{n}}\left|A_{1}(x, h)\right|^{2} \frac{d h}{|h|^{n+2 \theta}}\right)^{p / 2} d x\right)^{1 / p} \\
\leq & C\|K\|_{\infty} \sum_{k=1}^{n}\left\|L_{\theta}\left(\frac{\partial u}{\partial x_{k}}\right)\right\|_{L^{p}\left(\mathbf{R}^{n}\right)}\left(\int_{\{|z|>1\}}|z| \mu(d z)\right) .
\end{aligned}
$$

(2) The estimate of $A_{2}(x, h)$ : In this case, we have the inequality

$$
\begin{aligned}
& \left(\int_{\mathbf{R}^{n}}\left|A_{2}(x, h)\right|^{2} \frac{d h}{|h|^{n+2 \theta}}\right)^{1 / 2} \\
\leq & \int_{\{|z|>1\}}|u(x+z)-u(x)|\left(\int_{\mathbf{R}^{n}}|K(x+h, z)-K(x, z)|^{2} \frac{d h}{|h|^{n+2 \theta}}\right)^{1 / 2} \mu(d z) \\
\leq & H(\theta) \int_{\{|z|>1\}}|u(x+z)-u(x)| \mu(d z) .
\end{aligned}
$$


Hence, by applying Minkowskii's inequality for integrals and inequality (2.6) with $\gamma:=0$ we obtain that

$$
\begin{aligned}
& \left(\int_{\mathbf{R}^{n}}\left(\int_{\mathbf{R}^{n}}\left|A_{2}(x, h)\right|^{2} \frac{d h}{|h|^{n+2 \theta}}\right)^{p / 2} d x\right)^{1 / p} \\
\leq & C H(\theta)\|u\|_{L^{p}\left(\mathbf{R}^{n}\right)}\left(\int_{\{|z|>1\}}|z| \mu(d z)\right) .
\end{aligned}
$$

(3) The estimate of $A_{3}(x, h)$ : Since $p \geq 2$, it follows from definition $(2.1)$ that

$$
\left(\int_{\mathbf{R}^{n}}\left|A_{3}(x, h)\right|^{2} \frac{d h}{|h|^{n+2 \theta}}\right)^{1 / 2} \leq\|K\|_{\infty} \sum_{k=1}^{n} L_{\theta}\left(\frac{\partial u}{\partial x_{k}}(x)\right)\left(\int_{\{|z|>1\}}|z| \mu(d z)\right) .
$$

Hence we have, by Minkowskii's inequality for integrals,

$$
\begin{aligned}
& \left(\int_{\mathbf{R}^{n}}\left(\int_{\mathbf{R}^{n}}\left|A_{3}(x, h)\right|^{2} \frac{d h}{|h|^{n+2 \theta}}\right)^{p / 2} d x\right)^{1 / p} \\
\leq & \|K\|_{\infty} \sum_{j=1}^{n}\left\|L_{\theta}\left(\frac{\partial u}{\partial x_{j}}\right)\right\|_{L^{p}\left(\mathbf{R}^{n}\right)}\left(\int_{\mathbf{R}^{n}}|z| \mu(d z)\right) .
\end{aligned}
$$

(4) The estimate of $A_{4}(x, h)$ : In the last case we obtain that

$$
\begin{aligned}
& \left(\int_{\mathbf{R}^{n}}\left(\int_{\mathbf{R}^{n}}\left|A_{4}(x, h)\right|^{2} \frac{d h}{|h|^{n+2 \theta}}\right)^{p / 2} d x\right)^{1 / p} \\
\leq & H(\theta)\left(\int_{\{|z|>1\}}|z| \mu(d z)\right) \sum_{j=1}^{n}\left\|\frac{\partial u}{\partial x_{j}}\right\|_{L^{p}\left(\mathbf{R}^{n}\right)} .
\end{aligned}
$$

By applying Minkowskii's inequality for integrals to inequality (3.11), we obtain from inequalities (3.12) through (3.15) that

$$
\begin{aligned}
& \left\|L_{\theta}\left(S_{1} u\right)\right\|_{L^{p}\left(\mathbf{R}^{n}\right)} \\
\leq & C\left(\|K\|_{\infty}\left\|L_{\theta}\left(\frac{\partial u}{\partial x_{j}}\right)\right\|_{L^{p}\left(\mathbf{R}^{n}\right)}+H(\theta)\|u\|_{L^{p}\left(\mathbf{R}^{n}\right)}+H(\theta) \sum_{j=1}^{n}\left\|\frac{\partial u}{\partial x_{j}}\right\|_{L^{p}\left(\mathbf{R}^{n}\right)}\right) \\
& \times\left(\int_{\{|z|>1\}}|z| \mu(d z)\right) \\
\leq & C\left(\|K\|_{\infty}+H(\theta)\right) \\
& \times\left(\|u\|_{L^{p}\left(\mathbf{R}^{n}\right)}+\sum_{j=1}^{n}\left(\left\|L_{\theta}\left(\frac{\partial u}{\partial x_{j}}\right)\right\|_{L^{p}\left(\mathbf{R}^{n}\right)}+\left\|\frac{\partial u}{\partial x_{j}}\right\|_{L^{p}\left(\mathbf{R}^{n}\right)}\right)\right)
\end{aligned}
$$




$$
\times\left(\int_{\{|z|>1\}}|z| \mu(d z)\right) .
$$

However, we have, for $0<\theta<1$,

$$
\sum_{j=1}^{n}\left\|\frac{\partial u}{\partial x_{j}}\right\|_{L^{p}\left(\mathbf{R}^{n}\right)} \leq\|u\|_{H_{p}^{1}\left(\mathbf{R}^{n}\right)} \leq C\|u\|_{H_{p}^{\theta+1}\left(\mathbf{R}^{n}\right)}
$$

and, by Lemma 2.3,

$$
\sum_{j=1}^{n}\left\|L_{\theta}\left(\frac{\partial u}{\partial x_{j}}\right)\right\|_{L^{p}\left(\mathbf{R}^{n}\right)} \leq C \sum_{j=1}^{n}\left\|\frac{\partial u}{\partial x_{j}}\right\|_{H_{p}^{\theta}\left(\mathbf{R}^{n}\right)} \leq C\|u\|_{H_{p}^{1+\theta}\left(\mathbf{R}^{n}\right)} .
$$

Therefore, it follows from inequality (3.16) that

$$
\left\|L_{\theta}\left(S_{1} u\right)\right\|_{L^{p}\left(\mathbf{R}^{n}\right)} \leq C\left(\|K\|_{\infty}+H(\theta)\right)\|u\|_{H_{p}^{\theta+1}\left(\mathbf{R}^{n}\right)} \int_{\{|z|>1\}}|z| \mu(d z) .
$$

This proves the desired inequality (3.10).

(B) The estimate of $L_{\theta}\left(S_{2} u\right)$ : Secondly, we prove the inequality

$$
\begin{aligned}
& \left\|L_{\theta}\left(S_{2} u\right)\right\|_{L^{p}\left(\mathbf{R}^{n}\right)} \\
\leq & C\left(H(\theta)+\|K\|_{\infty}\right) \int_{\{0<|z| \leq 1\}}|z|^{2} \mu(d z) \cdot\|u\|_{H_{p}^{2+\theta}\left(\mathbf{R}^{n}\right)} .
\end{aligned}
$$

To do this, we write the difference

$$
S_{2} u(x+h)-S_{2} u(x)
$$

in the following form:

$$
S_{2} u(x+h)-S_{2} u(x):=B_{1}(x, h)-B_{2}(x, h),
$$

where

$$
\begin{aligned}
B_{1}(x, h)= & \int_{\{0<|z| \leq 1\}}(u(x+z+h)-u(x+z)-u(x+h)+u(x) \\
& \left.-\sum_{j=1}^{n} z_{j}\left(\frac{\partial u}{\partial x_{j}}(x+h)-\frac{\partial u}{\partial x_{j}}(x)\right)\right) K(x+h, z) \mu(d z)
\end{aligned}
$$

and

$$
\begin{aligned}
& B_{2}(x, h) \\
= & \int_{\{0<|z| \leq 1\}}\left(u(x+z)-u(x)-\sum_{j=1}^{n} z_{j} \frac{\partial u}{\partial x_{j}}(x)\right)(K(x+h, z)-K(x, z)) \mu(d z) .
\end{aligned}
$$


Then we have the inequality

$$
\begin{aligned}
L_{\theta}\left(S_{2} u\right)(x) & =\left(\int_{\mathbf{R}^{n}} \frac{\left|S_{2} u(x+h)-S_{2} u(x)\right|^{2}}{|h|^{n+2 \theta}} d h\right)^{1 / 2} \\
& \leq C\left(\int_{\mathbf{R}^{n}} \frac{\left|B_{1}(x, h)\right|^{2}}{|h|^{n+2 \theta}} d h+\int_{\mathbf{R}^{n}} \frac{\left|B_{2}(x, h)\right|^{2}}{|h|^{n+2 \theta}} d h\right) .
\end{aligned}
$$

First, if we let

$$
f_{t z}^{j}(x):=\frac{\partial u}{\partial x_{j}}(x+t z)-\frac{\partial u}{\partial x_{j}}(x)
$$

then it follows that

$$
\left|B_{1}(x, h)\right| \leq\|K\|_{\infty} \sum_{k=1}^{n} \int_{\{0<|z| \leq 1\}} \int_{0}^{1}|z|\left|f_{t z}^{k}(x+h)-f_{t z}^{k}(x)\right| d t \mu(d z) .
$$

Hence we have the inequality

$$
\left(\int_{\mathbf{R}^{n}} \frac{\left|B_{1}(x, h)\right|^{2}}{|h|^{n+2 \theta}} d h\right)^{1 / 2} \leq\|K\|_{\infty} \sum_{k=1}^{n} \int_{\{0<|z| \leq 1\}}|z| \int_{0}^{1} L_{\theta}\left(f_{t z}^{k}\right)(x) d t \mu(d z) .
$$

However, by applying inequality (2.6) with $\gamma:=\theta$ we obtain that

$$
\left\|L_{\theta}\left(f_{t z}^{j}\right)\right\|_{L^{p}\left(\mathbf{R}^{n}\right)} \leq C\left\|f_{t z}^{j}\right\|_{H_{p}^{\theta}\left(\mathbf{R}^{n}\right)} \leq C|t z|\left\|\frac{\partial u}{\partial x_{j}}\right\|_{H_{p}^{1+\theta}\left(\mathbf{R}^{n}\right)} \quad \text { for all } 0<t \leq 1 .
$$

Therefore, we have the inequality

$$
\begin{aligned}
& \left(\int_{\mathbf{R}^{n}}\left(\int_{\mathbf{R}^{n}} \frac{\left|B_{1}(x, h)\right|^{2}}{|h|^{n+2 \theta}} d h\right)^{p / 2} d x\right)^{1 / p} \\
\leq & C\|K\|_{\infty} \int_{\{0<|z| \leq 1\}}|z|^{2} \mu(d z) \sum_{j=1}^{n}\left\|\frac{\partial u}{\partial x_{j}}\right\|_{H_{p}^{1+\theta}\left(\mathbf{R}^{n}\right)} \\
\leq & C\|K\|_{\infty}\|u\|_{H_{p}^{2+\theta}\left(\mathbf{R}^{n}\right)} \int_{\{0<|z| \leq 1\}}|z|^{2} \mu(d z)
\end{aligned}
$$

On the other hand, we have the inequality

$$
\begin{aligned}
\left|B_{2}(x, h)\right| \leq & \sum_{j=1}^{n} \int_{0}^{1} \int_{\{0<|z| \leq 1\}}|z|\left|\frac{\partial u}{\partial x_{j}}(x+t z)-\frac{\partial u}{\partial x_{j}}(x)\right| \\
& \times|K(x+h, z)-K(x, z)| \mu(d z) d t .
\end{aligned}
$$

By the growth condition (1.4), it follows that

$$
\left(\int_{\mathbf{R}^{n}} \frac{\left|B_{2}(x, h)\right|^{2}}{|h|^{n+2 \theta}} d h\right)^{1 / 2}
$$




$$
\leq H(\theta) \sum_{j=1}^{n} \int_{\{0<|z| \leq 1\}}|z| \int_{0}^{1}\left|\frac{\partial u}{\partial x_{j}}(x+t z)-\frac{\partial u}{\partial x_{j}}(x)\right| \mu(d z) d t .
$$

By applying Minkowskii's inequality for integrals, we obtain from inequality (2.6) with $\gamma:=0$ that

$$
\begin{aligned}
& \left(\int_{\mathbf{R}^{n}}\left(\int_{\mathbf{R}^{n}} \frac{\left|B_{2}(x, h)\right|^{2}}{|h|^{n+2 \theta}} d h\right)^{p / 2} d x\right)^{1 / p} \\
\leq & C H(\theta) \sum_{k=1}^{n}\left\|\frac{\partial u}{\partial x_{k}}\right\|_{H_{p}^{1}\left(\mathbf{R}^{n}\right)} \int_{\{0<|z| \leq 1\}}|z|^{2} \mu(d z) \\
\leq & C H(\theta)\|u\|_{H_{p}^{2}\left(\mathbf{R}^{n}\right)} \int_{\{0<|z| \leq 1\}}|z|^{2} \mu(d z) .
\end{aligned}
$$

By applying Minkowskii's inequality for integrals to inequality (3.18), we obtain from inequalities (3.19) and (3.20) that

$$
\begin{aligned}
& \left\|L_{\theta}\left(S_{2} u\right)\right\|_{L^{p}\left(\mathbf{R}^{n}\right)} \\
\leq & C\left(\|K\|_{\infty}\|u\|_{H_{p}^{2+\theta}\left(\mathbf{R}^{n}\right)}+H(\theta)\|u\|_{H_{p}^{2}\left(\mathbf{R}^{n}\right)}\right) \int_{\{0<|z| \leq 1\}}|z|^{2} \mu(d z) \\
\leq & C\left(H(\theta)+\|K\|_{\infty}\right) \int_{\{0<|z| \leq 1\}}|z|^{2} \mu(d z) \cdot\|u\|_{H_{p}^{2+\theta}\left(\mathbf{R}^{n}\right)} .
\end{aligned}
$$

This proves the desired inequality (3.17).

Now the proof of inequality (3.3) (and hence that of Theorem 3.1) is complete.

\subsection{The case of a bounded domain}

We study the non-homogeneous boundary value problem (1.14) in the framework of generalized Sobolev spaces $H^{2+\theta, p}(\Omega)$ for $1<p<\infty$ and $0 \leq \theta<\theta_{0}$. To do so, we prove the following version of Theorem 3.1 with respect to the bounded domain $\Omega$ :

Theorem 3.2 Assume that the integral kernel $K(x, z)$ satisfies conditions (1.4), (1.5) and (1.6). Then the Lévy operator

$$
S: H_{p}^{2+\theta}(\Omega) \longrightarrow H_{p}^{\theta}(\Omega)
$$

is bounded for all $1<p<\infty$ and $0 \leq \theta \leq \theta_{0}$ (see Figure 3.1). Moreover, if $n<p<\infty$, then the Lévy operator

$$
S: H_{p}^{2+\theta}(\Omega) \longrightarrow H_{p}^{\theta}(\Omega)
$$

is compact for all $0 \leq \theta<\theta_{0}$. 


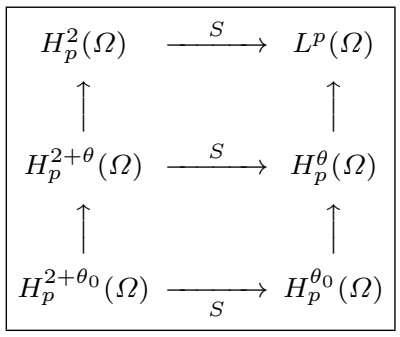

Fig. 3.1 The mapping properties of $S$ for $1<p<\infty$ and $0 \leq \theta \leq \theta_{0}$ in Theorem 3.2

Proof The proof of Theorem 3.2 is divided into four steps.

Step 1: By virtue of condition (1.5), the boundedness follows from Theorem 3.1 by restriction arguments:

$$
S: H_{p}^{2+\theta}(\Omega) \stackrel{E}{\longrightarrow} H_{p}^{2+\theta}\left(\mathbf{R}^{n}\right) \stackrel{S}{\longrightarrow} H_{p}^{\theta}\left(\mathbf{R}^{n}\right) \longrightarrow H_{p}^{\theta}(\Omega),
$$

where

$$
E: H_{p}^{2+\theta}(\Omega) \longrightarrow H_{p}^{2+\theta}\left(\mathbf{R}^{n}\right)
$$

is Seeley's extension operator (see [27], [1, Theorems 5.21 and 5.22]).

Step 2: In order to prove the compactness, we make use of an idea of Bony-Courrège-Priouret [7, Théorème XXI]. We show that the Lévy operator

$$
S: H_{p}^{2}(\Omega) \longrightarrow L^{p}(\Omega)
$$

is compact for $n<p<\infty$.

We recall that $K(x, z)=0$ if $x+z \notin \bar{\Omega}$. Hence, we can express $S u(x)$ as follows:

$$
S u(x)=\int_{M}\left(u(x+z)-u(x)-\sum_{j=1}^{n} z_{j} \frac{\partial u}{\partial x_{j}}(x)\right) K(x, z) \mu(d z),
$$

where

$$
M=\overline{\cup_{x \in \bar{\Omega}}\{\bar{\Omega}-x\}} .
$$

First, we take a smooth function $\chi$ in $C_{0}^{\infty}(\mathbf{R})$ such that

$$
\chi(t)= \begin{cases}1 & \text { if }|t| \leq 1 \\ 0 & \text { if }|t| \geq 2\end{cases}
$$

For every $0<\varepsilon<1$, we let

$$
\Phi_{\varepsilon}(z):=1-\chi\left(\frac{|z|}{\varepsilon}\right) .
$$

We remark that

$$
\Phi_{\varepsilon}(z)= \begin{cases}0 & \text { if }|z| \leq \varepsilon \\ 1 & \text { if }|z| \geq 2 \varepsilon\end{cases}
$$


Moreover, by Dini's theorem it follows that the sequence $\Phi_{\varepsilon}(x, z)$ converges uniformly to 1 for $z \neq 0$, as $\varepsilon \downarrow 0$.

Now we introduce a family of truncation operators given by the formula

$$
\begin{aligned}
& S_{\Phi_{\varepsilon}} u(x) \\
= & \int_{M}\left(u(x+z)-u(x)-\sum_{j=1}^{n} z_{j} \frac{\partial u}{\partial x_{j}}(x)\right) K(x, z) \Phi_{\varepsilon}(z) \mu(d z) \\
= & \int_{M \cap\{|z| \geq \varepsilon\}}\left(u(x+z)-u(x)-\sum_{j=1}^{n} z_{j} \frac{\partial u}{\partial x_{j}}(x)\right) K(x, z) \Phi_{\varepsilon}(z) \mu(d z) .
\end{aligned}
$$

By part (a) of Lemma 2.5, we can find a constant $C_{0}>0$ such that

$$
|K(x, z)-K(y, z)| \leq C_{0}|x-y|^{\theta_{0}} \quad \text { for all } x, y \in M \text { and almost all } z \in \mathbf{R}^{n} \text {. }
$$

Then it is easy to see that the Lévy operator

$$
S_{\Phi_{\varepsilon}}: C^{1}(\bar{\Omega}) \longrightarrow C(\bar{\Omega})
$$

is bounded. Indeed, we have, by the mean value theorem,

$$
\begin{aligned}
\left|S_{\Phi_{\varepsilon}} u(x)\right| \leq & \int_{M}\left|u(x+z)-u(x)-\sum_{j=1}^{n} z_{j} \frac{\partial u}{\partial x_{j}}(x)\right||K(x, z)| \Phi_{\varepsilon}(z) \mu(d z) \\
\leq & \int_{M \cap\{|z| \geq \varepsilon\}}|u(x+z)-u(x)||K(x, z)| \mu(d z) \\
& +\sum_{j=1}^{n} \int_{M \cap\{|z| \geq \varepsilon\}}\left|z_{j}\right||K(x, z)| \mu(d z)\left|\frac{\partial u}{\partial x_{j}}(x)\right| \\
\leq & C\|K\|_{\infty}\left(\int_{M \cap\{|z| \geq \varepsilon\}}|z| \mu(d z)\right)\|u\|_{C^{1}(\bar{\Omega})} \\
\leq & C\|K\|_{\infty}\left(\frac{1}{\varepsilon} \int_{\{\varepsilon \leq|z| \leq 1\}}|z|^{2} \mu(d z)+\int_{\{|z|>1\}}|z| \mu(d z)\right)\|u\|_{C^{1}(\bar{\Omega})} \\
& \quad \text { for all } x \in \bar{\Omega} .
\end{aligned}
$$

This proves that $S_{\Phi_{\varepsilon}} u \in C(\bar{\Omega})$ for all $u \in C^{1}(\bar{\Omega})$, since the function

$$
\bar{\Omega} \ni x \longmapsto K(x, z)
$$

is continuous for almost all $z \in \mathbf{R}^{n}$.

Since the embedding

$$
H_{p}^{2}(\Omega) \hookrightarrow \hookrightarrow C^{1}(\bar{\Omega})
$$

is compact for $n<p<\infty$ (see the Rellich-Kondrachov theorem [1, Theorem 6.3 and Paragraph 7.32]) and since the embedding

$$
C(\bar{\Omega}) \longrightarrow L^{p}(\Omega)
$$


is continuous, we obtain that the Lévy operator

$$
S_{\Phi_{\varepsilon}}: H_{p}^{2}(\Omega) \longrightarrow L^{p}(\Omega)
$$

is compact. The situation can be visualized as follows:

$$
S_{\Phi_{\varepsilon}}: H_{p}^{2}(\Omega) \underset{\text { compactly }}{\hookrightarrow \hookrightarrow} C^{1}(\bar{\Omega}) \stackrel{S_{\Phi_{\varepsilon}}}{\longrightarrow} C(\bar{\Omega}) \longrightarrow L^{p}(\Omega)
$$

On the other hand, we obtain from inequality (3.2) that

$$
\begin{aligned}
& \qquad S_{\Phi_{\varepsilon}} u \|_{L^{p}(\Omega)} \\
& \leq C\|K\|_{\infty}\left(\int_{\{|z|>1\}}|z| \chi\left(\frac{|z|}{\varepsilon}\right) \mu(d z)+\int_{\{0<|z| \leq 1\}}|z|^{2} \chi\left(\frac{|z|}{\varepsilon}\right) \mu(d z)\right) \\
& \quad \times\|u\|_{H_{p}^{2}(\Omega)} \text { for all } u \in H_{p}^{2}(\Omega) \text { and } 0<\varepsilon<1 .
\end{aligned}
$$

Furthermore, it follows from an application of Lebesgue's dominated convergence theorem that

$$
S_{\Phi_{\varepsilon}} \longrightarrow S \quad \text { as } \varepsilon \downarrow 0
$$

with respect to the operator norm in the space $\mathcal{L}\left(H_{p}^{2}(\Omega), L^{p}(\Omega)\right)$ of bounded linear operators on $H_{p}^{2}(\Omega)$ into $L^{p}(\Omega)$. Indeed, just as in the proof of inequality (3.2) we obtain that

$$
\begin{aligned}
& \left\|S u-S_{\Phi_{\varepsilon}} u\right\|_{L^{p}(\Omega)} \\
\leq & C\|K\|_{\infty}\left(\int_{\{|z|>1\}}|z| \chi\left(\frac{|z|}{\varepsilon}\right) \mu(d z)+\int_{\{0<|z| \leq 1\}}|z|^{2} \chi\left(\frac{|z|}{\varepsilon}\right) \mu(d z)\right) \\
& \times\|u\|_{H_{p}^{2}(\Omega)} \text { for all } u \in H_{p}^{2}(\Omega) .
\end{aligned}
$$

However, by the moment condition (1.6) it follows from Lebesgue's dominated convergence theorem that

$$
\lim _{\varepsilon \downarrow 0}\left(\int_{\{|z|>1\}}|z| \chi\left(\frac{|z|}{\varepsilon}\right) \mu(d z)+\int_{\{0<|z| \leq 1\}}|z|^{2} \chi\left(\frac{|z|}{\varepsilon}\right) \mu(d z)\right)=0 .
$$

Therefore, by applying [43, p. 278, Theorem] (or [26, Theorem 4.11]) we obtain from assertions (3.21) and (3.22) that the Lévy operator

$$
S: H_{p}^{2}(\Omega) \longrightarrow L^{p}(\Omega)
$$

is compact for $n<p<\infty$.

Step 3: Theorem 3.1 tells us that the Lévy operator

$$
S: H_{p}^{2+\theta}(\Omega) \longrightarrow H_{p}^{\theta}(\Omega)
$$

is bounded for all $1<p<\infty$ and $0 \leq \theta \leq \theta_{0}$. Moreover, we obtain from Step 2 that the Lévy operator

$$
S: H_{p}^{2}(\Omega) \longrightarrow L^{p}(\Omega)
$$


is compact for $n<p<\infty$.

Step 4: Finally, in order to prove Theorem 3.2 we make use of the following result concerning the complex interpolation of compact linear operators, due to Cwikel-Kalton [10, Theorem 10]):

Theorem 3.3 (Cwikel-Kalton) Let $\left(X_{0}, X_{1}\right)$ be a Banach couple such that $X_{0}$ is reflexive and is given by the formula

$$
X_{0}=\left[X_{1}, W\right]_{\alpha}
$$

for some $0<\alpha<1$ and some Banach space $W$ which forms a Banach couple with $X_{1}$. Here $\left[W, X_{1}\right]_{\alpha}$ is Calderón's complex method (see [8]). Assume that the operator

$$
T: X_{j} \longrightarrow Y_{j}
$$

is bounded for $j=0,1$, and further that the operator

$$
T: X_{0} \longrightarrow Y_{0}
$$

is compact. Then the operator

$$
T: X_{\gamma}=\left[X_{0}, X_{1}\right]_{\gamma} \longrightarrow Y_{\gamma}=\left[Y_{0}, Y_{1}\right]_{\gamma}
$$

is compact for each $0<\gamma<1$ (see Figure 3.2).

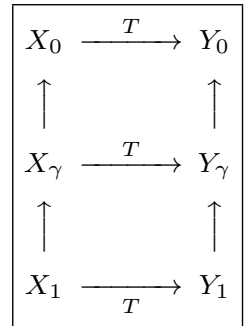

Fig. 3.2 The mapping properties of $T$ for $0<\gamma<1$ in Theorem 3.3

First, it is known (see $[1$, Chapter 3 , Theorem 3.6] and $[1$, p. 250]) that $H^{2, p}(\Omega)$ is reflexive and further (see [36, p. 88, Theorem] and [37, p. 44, Theorem]) that

$$
H_{p}^{2}(\Omega)=\left[H_{p}^{2+\theta}(\Omega), L^{p}(\Omega)\right]_{\alpha}, \quad \alpha=\frac{2}{2+\theta} .
$$

Therefore, by applying Theorem 3.3 with

$$
\begin{aligned}
& X_{1}:=H_{p}^{2+\theta_{0}}(\Omega), \quad W:=L^{p}(\Omega), \\
& X_{0}:=H_{p}^{2}(\Omega)=\left[H_{p}^{2+\theta_{0}}(\Omega), L^{p}(\Omega)\right]_{\alpha}, \quad \alpha=\frac{2}{2+\theta_{0}},
\end{aligned}
$$




$$
Y_{0}:=L^{p}(\Omega), \quad Y_{1}:=H_{p}^{\theta_{0}}(\Omega),
$$

we obtain that the Lévy operator

$$
S: H_{p}^{2+\theta}(\Omega) \longrightarrow H_{p}^{\theta}(\Omega)
$$

is compact for all $0 \leq \theta<\theta_{0}$ and $n<p<\infty$.

Now the proof of Theorem 3.2 is complete.

\section{Proof of Theorem 1.1}

This section is devoted to the proof of Theorem 1.1. The proof is divided into two Subsections 4.1 and 4.2, due to its length.

4.1 The differential operator case

First, we consider the case where $S \equiv 0$ and $T \equiv 0$. Namely, we consider the boundary value problem

$$
\begin{cases}A u=f & \text { in } \Omega, \\ \Lambda u=\mu\left(x^{\prime}\right) \frac{\partial u}{\partial \mathbf{n}}+\gamma\left(x^{\prime}\right) u=\varphi & \text { on } \partial \Omega\end{cases}
$$

in the framework of Sobolev spaces $H_{p}^{2+\theta}(\Omega)$ and Besov spaces $B_{p, p, \star}^{1+\theta-1 / p}(\partial \Omega)$.

The next theorem is proved in Taira [30, Theorem 1] and [33, Theorem 1.1]. A more general result in the framework of spaces of Besov-Triebel-Lizorkin type $F_{p, q}^{s}$ and $B_{p, q}^{s}$ is given by Runst [22].

Theorem 4.1 Let $1<p<\infty$. If condition (1.13) is satisfied, then the mapping

$$
(A, \Lambda): H_{p}^{2+\theta}(\Omega) \longrightarrow H_{p}^{\theta}(\Omega) \oplus B_{p, p, \star}^{1+\theta-1 / p}(\partial \Omega)
$$

is an algebraic and topological isomorphism for all $0 \leq \theta<\theta_{0}$. In particular, there exists a unique solution $u \in H_{p}^{2+\theta}(\Omega)$ of the problem (4.1) for any $f \in$ $H_{p}^{\theta}(\Omega)$ and any $\varphi \in B_{p, p, \star}^{1+\theta-1 / p}(\partial \Omega)$,

4.2 End of Proof of Theorem 1.1

First, we have the formula

$$
(W, \Lambda)=(A+S, \Lambda)=(A, \Lambda)+(S, 0) .
$$

However, by Theorem 4.1 it follows that

$$
\operatorname{ind}(A, \Lambda)=0 \text {. }
$$


Moreover, by using Theorem 3.2 we obtain that the Lévy operator

$$
S: H_{p}^{2+\theta}(\Omega) \longrightarrow H_{p}^{\theta}(\Omega)
$$

is compact for all $0 \leq \theta<\theta_{0}$. Namely, the operator

$$
(W, \Lambda)=(A+S, \Lambda)=(A, \Lambda)+(S, 0)
$$

is a compact perturbation of the operator $(A, \Lambda)$.

Hence we have, by assertion (4.2),

$$
\text { ind }(W, \Lambda)=\operatorname{ind}(A, \Lambda)=0 \text {, }
$$

since the index is stable under compact perturbations (see [15, Theorem 2.6], [26, Theorem 5.10]).

Therefore, the proof of Theorem 1.1 is complete if we prove that the operator

$$
(W, \Lambda): H_{p}^{2+\theta}(\Omega) \longrightarrow H_{p}^{\theta}(\Omega) \oplus B_{p, p, \star}^{1+\theta-1 / p}(\partial \Omega)
$$

is injective for all $0 \leq \theta<\theta_{0}$.

In this way, Theorem 1.1 follows by combining assertion (4.3) and the following uniqueness theorem:

Theorem 4.2 (the uniqueness theorem) Let $0<\theta<1$ and $n<p<\infty$. Assume that $c(x) \leq 0$ in $\Omega$, but $c(x) \not \equiv 0$ in $\Omega$ and further that condition (1.13) is satisfied. If $u \in H_{p}^{2+\theta}(\Omega)$ is a solution of the homogeneous boundary value problem

$$
\begin{cases}W u=0 & \text { in } \Omega, \\ \Lambda u=0 & \text { on } \partial \Omega,\end{cases}
$$

then it follows that

$$
u=0 \quad \text { on } \bar{\Omega} \text {. }
$$

Proof (i) First, we consider the case where $u$ is a constant $M$ in $\Omega$. Then we have the formula

$$
0=W u(x)=A u(x)+S u(x)=M c(x) \text { in } \Omega .
$$

This proves that $M=0$, since $c(x) \not \equiv 0$ in $\Omega$.

(ii) Secondly, we consider the case where $u$ is not a constant in $\Omega$. Our proof is based on a reduction to absurdity. If (necessarily replacing $u(x)$ by $-u(x)$ ), we may assume that there exists a point $x_{0}$ of the closure $\bar{\Omega}$ such that

$$
u\left(x_{0}\right)=\max _{x \in \bar{\Omega}} u(x)>0 .
$$

If $x_{0}$ is an interior point, then it follows from an application of the strong maximum principle (Theorem 6.2 ) that $u(x)$ is a constant in $\Omega$. This is a contradiction. 
Now we assume that there exists a boundary point $x_{0}$ such that

$$
\left\{\begin{array}{l}
u\left(x_{0}\right)=\max _{x \in \bar{\Omega}} u(x)>0, \\
u(y)<u\left(x_{0}\right) \text { for all } y \in \Omega .
\end{array}\right.
$$

Then, by applying the Hopf boundary point lemma (Lemma 6.1) we obtain that

Hence we have the formula

$$
\frac{\partial u}{\partial \mathbf{n}}\left(x_{0}\right)<0
$$

$$
0=\Lambda u\left(x_{0}\right)=\mu\left(x_{0}\right) \frac{\partial u}{\partial \mathbf{n}}\left(x_{0}\right)+\gamma\left(x_{0}\right) u\left(x_{0}\right) .
$$

However, since we have the assertions

$$
\begin{aligned}
& \frac{\partial u}{\partial \mathbf{n}}\left(x_{0}\right)<0, \\
& u\left(x_{0}\right)=\max _{x \in \bar{\Omega}} u(x)>0,
\end{aligned}
$$

it follows from condition (1.13) that

$$
\mu\left(x_{0}\right) \frac{\partial u}{\partial \mathbf{n}}\left(x_{0}\right)+\gamma\left(x_{0}\right) u\left(x_{0}\right)<0 .
$$

This contradicts formula (4.4).

The proof of Theorem 4.2 is complete.

\section{Proof of Theorem 1.2}

This section is devoted to the proof of Theorem 1.2. The proof is divided into two Subsections 5.1 and 5.2, due to its length.

5.1 Mapping properties of Lévy-Ventcel' operators

In this subsection, we study the Lévy-Ventcel' boundary operator

$$
\begin{aligned}
T u\left(x^{\prime}\right)=\gamma_{0}(T u) & =\int_{\mathbf{R}^{n} \backslash\{0\}}\left(u\left(x^{\prime}+z\right)-u\left(x^{\prime}\right)\right) J\left(x^{\prime}, z\right) \nu(d z) \\
\text { for } x^{\prime} & \in \partial \Omega,
\end{aligned}
$$

where $\gamma_{0}$ is the trace operator.

Our main result of this subsection is stated as follows:

Theorem 5.1 Assume that conditions (1.9) through (1.12) are satisfied. Then the Lévy-Ventcel' boundary operator

$$
\gamma_{0} T: H_{p}^{2+\theta}\left(\mathbf{R}^{n}\right) \longrightarrow B_{p, p}^{1+\theta-1 / p}\left(\mathbf{R}^{n-1}\right)
$$

is bounded for all $1<p<\infty$ and $0 \leq \theta \leq \theta_{1}$. 
Proof We remark that trace operator

$$
\gamma_{0}: H_{p}^{1+\theta}\left(\mathbf{R}^{n}\right)=F_{p, 2}^{1+\theta}\left(\mathbf{R}^{n}\right) \longrightarrow F_{p, p}^{1+\theta-1 / p}\left(\mathbf{R}^{n-1}\right)=B_{p, p}^{1+\theta-1 / p}\left(\mathbf{R}^{n-1}\right)
$$

is continuous for all $1<p<\infty$. Here the space $F_{p, q}^{s}\left(\mathbf{R}^{n}\right)$ are called TriebelLizorkin spaces (see [37, Theorem 4.4.2]). Hence we are reduced to the study of the integro-differential operator

$$
T u(x)=\int_{\mathbf{R}^{n} \backslash\{0\}}(u(x+z)-u(x)) J(x, z) \nu(d z) \quad \text { for } x \in \mathbf{R}^{n}
$$

under the moment condition (1.12).

The proof of Theorem 5.1 is divided into two steps.

Step (i): First, we find that the integro-differential operator

$$
T: H_{p}^{1+\theta}\left(\mathbf{R}^{n}\right) \longrightarrow H_{p}^{\theta}\left(\mathbf{R}^{n}\right)
$$

is bounded for all $0 \leq \theta<1$, just as in the proof of Theorem 3.1. In fact, we can obtain the following inequalities (see the inequalities (3.2) and (3.3)):

$$
\begin{aligned}
& \|T u\|_{L^{p}\left(\mathbf{R}^{n}\right)} \\
\leq & \|J\|_{\infty}\left\{\int_{\{0<|z| \leq 1\}}|z| \nu(d z) \cdot\|\nabla u\|_{L^{p}\left(\mathbf{R}^{n}\right)}+2 \int_{\{|z|>1\}} \nu(d z) \cdot\|u\|_{L^{p}\left(\mathbf{R}^{n}\right)}\right\} \\
\leq & C\|J\|_{\infty}\left(\int_{\{0<|z| \leq 1\}}|z| \nu(d z)+\int_{\{|z|>1\}} \nu(d z)\right)\|u\|_{H_{p}^{1}\left(\mathbf{R}^{n}\right)}
\end{aligned}
$$

and (see Remark 1.1)

$$
\begin{aligned}
& \left\|L_{\theta}(T u)\right\|_{L^{p}\left(\mathbf{R}^{n}\right)} \\
\leq & C\left(\|J\|_{\infty}+\left\|\nabla_{x} J\right\|_{\infty}\right)\left(\int_{\{0<|z| \leq 1\}}|z| \nu(d z)+\int_{\{|z|>1\}} \nu(d z)\right)\|u\|_{H_{p}^{1+\theta}\left(\mathbf{R}^{n}\right)} \\
& \text { for all } 0 \leq \theta<1 .
\end{aligned}
$$

Step (ii): Secondly, we show that the integro-differential operator

$$
T: H_{p}^{2+\theta}\left(\mathbf{R}^{n}\right) \longrightarrow H_{p}^{1+\theta}\left(\mathbf{R}^{n}\right)
$$

is bounded for all $0 \leq \theta \leq \theta_{1}$.

To do so, we make use of the formulas

$$
\begin{aligned}
\frac{\partial}{\partial x_{i}}(T u(x))= & \int_{\mathbf{R}^{n} \backslash\{0\}}\left(\frac{\partial u}{\partial x_{i}}(x+z)-\frac{\partial u}{\partial x_{i}}(x)\right) J(x, z) \nu(d z) \\
& +\int_{\mathbf{R}^{n} \backslash\{0\}}(u(x+z)-u(x)) \frac{\partial J}{\partial x_{i}}(x, z) \nu(d z) \\
:= & A_{i}(x)+B_{i}(x) \quad \text { for } 1 \leq i \leq n .
\end{aligned}
$$

Hence, it suffices to show that we have, for $1 \leq i \leq n$,

$$
A_{i}, B_{i} \in L^{p}\left(\mathbf{R}^{n}\right),
$$




$$
L_{\theta}\left(A_{i}\right), L_{\theta}\left(B_{i}\right) \in L^{p}\left(\mathbf{R}^{n}\right) .
$$

First, we can prove the following inequalities (see the inequality (5.1)):

$$
\begin{aligned}
& -\left\|A_{i}\right\|_{L^{p}\left(\mathbf{R}^{n}\right)} \\
\leq & C\|J\|_{\infty}\left(\int_{\{0<|z| \leq 1\}}|z| \nu(d z)+\int_{\{|z|>1\}} \nu(d z)\right)\|\nabla u\|_{H_{p}^{1}\left(\mathbf{R}^{n}\right)} . \\
& -\left\|B_{i}\right\|_{L^{p}\left(\mathbf{R}^{n}\right)} \\
\leq & C\left\|\nabla_{x} J\right\|_{\infty}\left(\int_{\{0<|z| \leq 1\}}|z| \nu(d z)+\int_{\{|z|>1\}} \nu(d z)\right)\|u\|_{H_{p}^{1}\left(\mathbf{R}^{n}\right)} .
\end{aligned}
$$

Therefore, by using inequalities (5.5) we obtain from formula (5.3) that

$$
\begin{aligned}
& \|T u\|_{H_{p}^{1}\left(\mathbf{R}^{n}\right)} \\
\leq & C\left(\|J\|_{\infty}+\left\|\nabla_{x} J\right\|_{\infty}\right)\left(\int_{\{0<|z| \leq 1\}}|z| \nu(d z)+\int_{\{|z|>1\}} \nu(d z)\right)\|u\|_{H_{p}^{2}\left(\mathbf{R}^{n}\right)} .
\end{aligned}
$$

Moreover, we can prove the following inequalities for all $0 \leq \theta \leq \theta_{1}$ (see the inequality (5.2)):

$$
\begin{aligned}
& \left\|L_{\theta}\left(A_{i}\right)\right\|_{L^{p}\left(\mathbf{R}^{n}\right)} \\
\leq & C\|J\|_{\infty}\left(\int_{\{0<|z| \leq 1\}}|z| \nu(d z)+\int_{\{|z|>1\}} \nu(d z)\right)\|\nabla u\|_{H_{p}^{1}\left(\mathbf{R}^{n}\right)} \\
\leq & C\|J\|_{\infty}\left(\int_{\{0<|z| \leq 1\}}|z| \nu(d z)+\int_{\{|z|>1\}} \nu(d z)\right)\|u\|_{H_{p}^{2}\left(\mathbf{R}^{n}\right)} \\
\quad & \text { for } 1 \leq i \leq n,
\end{aligned}
$$

and (see Remark 1.1)

$$
\begin{aligned}
& \left\|L_{\theta}\left(B_{i}\right)\right\|_{L^{p}\left(\mathbf{R}^{n}\right)} \\
& \leq C\left(\left\|\nabla_{x} J\right\|_{\infty}+G(\theta)\right)\left(\int_{\{0<|z| \leq 1\}}|z| \nu(d z)+\int_{\{|z|>1\}} \nu(d z)\right)\|u\|_{H_{p}^{1+\theta}\left(\mathbf{R}^{n}\right)} .
\end{aligned}
$$

Therefore, the desired assertions (5.4b) follow from inequalities (5.7) and (5.8).

The proof of Theorem 5.1 is complete.

By combining Theorem 5.1 with the Rellich-Kondrachov theorem ([1, Theorem 6.3 and Paragraph 7.32]) and the trace theorem ([37, Theorem 4.4.2]), we can obtain the following:

Theorem 5.2 Assume that conditions (1.9) through (1.12) are satisfied. Then the Lévy-Ventcel' boundary operator

$$
\gamma_{0} T: H_{p}^{2+\theta}(\Omega) \longrightarrow B_{p, p}^{1+\theta-1 / p}(\partial \Omega)
$$

is compact for all $n<p<\infty$ and $0 \leq \theta<\theta_{1}$. 
Proof The proof of Theorem 5.2 is divided into four steps.

Step 1: Just as in the proof of Theorem 3.2, we introduce a family of truncation operators given by the formula

$$
\begin{aligned}
T_{\Phi_{\varepsilon}} u(x) & =\int_{\mathbf{R}^{n} \backslash\{0\}}(u(x+z)-u(x)) J(x, z) \Phi_{\varepsilon}(z) \nu(d z) \\
& =\int_{\{|z| \geq \varepsilon\}}(u(x+z)-u(x)) J(x, z) \Phi_{\varepsilon}(z) \nu(d z) .
\end{aligned}
$$

Then it is easy to see that the operator

$$
T_{\Phi_{\varepsilon}}: C^{1}(\bar{\Omega}) \longrightarrow C^{1}(\bar{\Omega})
$$

is bounded. Indeed, we have the inequality

$$
\begin{aligned}
\left|T_{\Phi_{\varepsilon}} u(x)\right| & \leq 2\|J\|_{\infty}\|u\|_{C(\bar{\Omega})}\left(\int_{\{\varepsilon \leq|z| \leq 1\}} \nu(d z)+\int_{\{|z|>1\}} \nu(d z)\right) \\
& \leq 2\|J\|_{\infty}\left(\frac{1}{\varepsilon} \int_{\{\varepsilon \leq|z| \leq 1\}}|z| \nu(d z)+\int_{\{|z|>1\}} \nu(d z)\right)\|u\|_{C(\bar{\Omega})}
\end{aligned}
$$

for all $x \in \bar{\Omega}$.

Hence, by virtue of inequality (5.10) it follows from an application of the Lebesgue dominated convergence theorem that

$$
T_{\Phi_{\varepsilon}} u \in C(\bar{\Omega}) \text { for all } u \in C(\bar{\Omega}),
$$

since the function

$$
\bar{\Omega} \ni x \longmapsto J(x, z)
$$

is continuous for almost all $z \in \mathbf{R}^{n}$.

Moreover, we have the formulas

$$
\begin{aligned}
\frac{\partial}{\partial x_{i}}\left(T_{\Phi_{\varepsilon}} u(x)\right)= & \int_{\{|z| \geq \varepsilon\}}\left(\frac{\partial u}{\partial x_{i}}(x+z)-\frac{\partial u}{\partial x_{i}}(x)\right) J(x, z) \Phi_{\varepsilon}(z) \nu(d z) \\
& \quad+\int_{\{|z| \geq \varepsilon\}}(u(x+z)-u(x)) \frac{\partial J}{\partial x_{i}}(x, z) \Phi_{\varepsilon}(z) \nu(d z) \\
:= & C_{i}(x)+D_{i}(x) \text { for } 1 \leq i \leq n .
\end{aligned}
$$

However, we have the inequalities (see the inequality (5.1))

$$
\begin{aligned}
\left|C_{i}(x)\right| \leq 2 & \|J\|_{\infty}\left(\frac{1}{\varepsilon} \int_{\{\varepsilon \leq|z| \leq 1\}}|z| \nu(d z)+\int_{\{|z|>1\}} \nu(d z)\right)\|\nabla u\|_{C(\bar{\Omega})} \\
& \text { for all } x \in \bar{\Omega},
\end{aligned}
$$

and

$$
\left|D_{i}(x)\right| \leq 2\left\|\nabla_{x} J\right\|_{\infty}\left(\frac{1}{\varepsilon} \int_{\{\varepsilon \leq|z| \leq 1\}}|z| \nu(d z)+\int_{\{|z|>1\}} \nu(d z)\right)\|u\|_{C(\bar{\Omega})}
$$


for all $x \in \bar{\Omega}$.

Therefore, by using inequalities (5.12) and (5.13) we obtain from formula (5.11) that

$$
\begin{aligned}
& \left|\frac{\partial}{\partial x_{i}}\left(T_{\Phi_{\varepsilon}} u(x)\right)\right| \leq\left|C_{i}(x)\right|+\left|D_{i}(x)\right| \\
\leq & 2\left(\|J\|_{\infty}+\left\|\nabla_{x} J\right\|_{\infty}\right)\left(\frac{1}{\varepsilon} \int_{\{\varepsilon \leq|z| \leq 1\}}|z| \nu(d z)+\int_{\{|z|>1\}} \nu(d z)\right)\|u\|_{C^{1}(\bar{\Omega})}
\end{aligned}
$$

for all $x \in \bar{\Omega}$.

By virtue of inequality (5.14), it follows from an application of Lebesgue's dominated convergence theorem that $T_{\Phi_{\varepsilon}} u \in C^{1}(\bar{\Omega})$ for all $u \in C^{1}(\bar{\Omega})$, since the functions

$$
\bar{\Omega} \ni x \longmapsto J(x, z)
$$

and

$$
\bar{\Omega} \ni x \longmapsto \frac{\partial J}{\partial x_{i}}(x, z), \quad 1 \leq i \leq n,
$$

are all continuous for almost all $z \in \mathbf{R}^{n}$ (see part (a) of Lemma 2.5).

Step 2: Summing up, we find that the operator

$$
T_{\Phi_{\varepsilon}}: H_{p}^{2}(\Omega) \longrightarrow H_{p}^{1}(\Omega)
$$

is compact for $n<p<\infty$. More precisely, the situation can be visualized as follows:

$$
H_{p}^{2}(\Omega): \underset{\text { compactly }}{\hookrightarrow} C^{1}(\bar{\Omega}) \stackrel{T_{\Phi_{\bar{g}}}}{\longrightarrow} C^{1}(\bar{\Omega}) \longrightarrow H_{p}^{1}(\Omega) .
$$

Step 3: Furthermore, it follows from an application of Lebesgue's dominated convergence theorem that

$$
T_{\Phi_{\varepsilon}} \longrightarrow T \quad \text { as } \varepsilon \downarrow 0
$$

with respect to the operator norm in the space $\mathcal{L}\left(H_{p}^{2}(\Omega), H_{p}^{1}(\Omega)\right)$ of bounded linear operators on $H_{p}^{2}(\Omega)$ into $H_{p}^{1}(\Omega)$.

Indeed, just as in the proof of inequality (5.1) we obtain that

$$
\begin{aligned}
& \left\|T u-T_{\Phi_{\varepsilon}} u\right\|_{L^{p}(\Omega)} \\
= & \left\|\int_{\mathbf{R}^{n} \backslash\{0\}}(u(x+z)-u(x)) J(x, z) \chi\left(\frac{|z|}{\varepsilon}\right) \nu(d z)\right\|_{L^{p}(\Omega)} \\
\leq & C\|J\|_{\infty}\left(\int_{\{0<|z| \leq 1\}}|z| \chi\left(\frac{|z|}{\varepsilon}\right) \nu(d z)+\int_{\{|z|>1\}} \chi\left(\frac{|z|}{\varepsilon}\right) \nu(d z)\right)\|u\|_{H_{p}^{1}\left(\mathbf{R}^{n}\right)} .
\end{aligned}
$$

Moreover, we have, by formulas (5.11) for $1 \leq i \leq n$,

$$
\left\|\nabla\left(T u-T_{\Phi_{\varepsilon}} u\right)\right\|_{L^{p}(\Omega)}
$$




$$
\begin{aligned}
& \leq C\|J\|_{\infty}\left(\int_{\{0<|z| \leq 1\}}|z| \chi\left(\frac{|z|}{\varepsilon}\right) \nu(d z)+\int_{\{|z|>1\}} \chi\left(\frac{|z|}{\varepsilon}\right) \nu(d z)\right)\|u\|_{H_{p}^{2}\left(\mathbf{R}^{n}\right)} \\
& +C\left\|\nabla_{x} J\right\|_{\infty}\left(\int_{\{0<|z| \leq 1\}}|z| \chi\left(\frac{|z|}{\varepsilon}\right) \nu(d z)+\int_{\{|z|>1\}} \chi\left(\frac{|z|}{\varepsilon}\right) \nu(d z)\right)\|u\|_{H_{p}^{1}\left(\mathbf{R}^{n}\right)} .
\end{aligned}
$$

However, by the moment condition (1.12) it follows from Lebesgue's dominated convergence theorem that

$$
\lim _{\varepsilon \downarrow 0}\left\{\int_{\{0<|z| \leq 1\}}|z| \chi\left(\frac{|z|}{\varepsilon}\right) \nu(d z)+\int_{\{|z|>1\}} \chi\left(\frac{|z|}{\varepsilon}\right) \nu(d z)\right\}=0 .
$$

Therefore, the desired assertion (5.15) follows by combining inequalities (5.16) and (5.17).

By using [43, p. 278, Theorem] (or [26, Theorem 4.11]), we find that the operator

$$
T: H_{p}^{2}(\Omega) \longrightarrow H_{p}^{1}(\Omega)
$$

is compact for $n<p<\infty$.

Step 4: Finally, by applying Theorem 3.3 with

$$
\begin{aligned}
& X_{1}:=H_{p}^{2+\theta_{1}}(\Omega), \quad W:=L^{p}(\Omega), \\
& X_{0}:=H_{p}^{2}(\Omega)=\left[H_{p}^{2+\theta_{1}}(\Omega), L^{p}(\Omega)\right]_{\alpha}, \quad \alpha=\frac{2}{2+\theta_{1}}, \\
& Y_{0}:=H_{p}^{1}(\Omega), \quad Y_{1}:=H_{p}^{1+\theta_{1}}(\Omega),
\end{aligned}
$$

we obtain that the operator

$$
T: H_{p}^{2+\theta}(\Omega) \longrightarrow H_{p}^{1+\theta}(\Omega)
$$

is compact for all $0 \leq \theta<\theta_{1}$ and $n<p<\infty$ (see Figure 5.1 below).

Now the proof of Theorem 5.2 is complete.

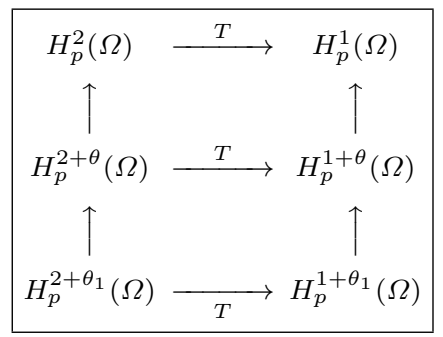

Fig. 5.1 The mapping properties of $T$ for $0 \leq \theta<\theta_{1}$ and $n<p<\infty$ in Theorem 5.2 
5.2 End of Proof of Theorem 1.2

First, we remark that the index of the mapping

$$
(W, \Lambda): H_{p}^{2+\theta}(\Omega) \longrightarrow H_{p}^{\theta}(\Omega) \oplus B_{p, p}^{1+\theta-1 / p}(\partial \Omega)
$$

is equal to zero:

$$
\text { ind }(W, \Lambda)=0
$$

under the condition

$$
\mu\left(x^{\prime}\right)>0 \quad \text { on } \partial \Omega \text {. }
$$

However, by using Theorem 5.2 we obtain that the Lévy-Ventcel' boundary operator

$$
\gamma_{0} T: H_{p}^{2+\theta}(\Omega) \longrightarrow B_{p, p}^{1+\theta-1 / p}(\partial \Omega)
$$

is compact for all $1<p<\infty$ and $0 \leq \theta<\theta_{1}$. Namely, the operator

$$
(W, L)=\left(W, \Lambda+\gamma_{0} T\right)=(W, \Lambda)+\left(0, \gamma_{0} T\right)
$$

is a compact perturbation of the operator $(W, \Lambda)$.

Hence we have, by the assertion (4.3),

$$
\text { ind }(W, L)=\operatorname{ind}(W, \Lambda)=0 \text {, }
$$

since the index is stable under compact perturbations (see [15, Theorem 2.6], [26, Theorem 5.10]).

Therefore, the proof of Theorem 1.2 is complete if we prove that the operator

$$
(W, L): H_{p}^{2+\theta}(\Omega) \longrightarrow H_{p}^{\theta}(\Omega) \oplus B_{p, p}^{1+\theta-1 / p}(\partial \Omega)
$$

is injective for $0 \leq \theta<\min \left\{\theta_{0}, \theta_{1}\right\}$.

In this way, Theorem 1.2 follows by combining assertion (5.18) and the following uniqueness theorem:

Theorem 5.3 (the uniqueness theorem) Let $n<p<\infty$. Assume that the following transversality condition is satisfied:

$$
\mu\left(x^{\prime}\right)-\gamma\left(x^{\prime}\right)+\int_{\left\{x^{\prime}+z \in \Omega\right\}} J\left(x^{\prime}, z\right) \nu(d z)>0 \quad \text { on } \partial \Omega .
$$

If $u \in H_{p}^{2+\theta}(\Omega)$ is a solution of the homogeneous boundary value problem

$$
\begin{cases}W u=(A+S) u=0 & \text { in } \Omega, \\ L u=\left(\Lambda+\gamma_{0} T\right) u=0 & \text { on } \partial \Omega\end{cases}
$$

then it follows that

$$
u=0 \quad \text { on } \bar{\Omega} \text {. }
$$


Proof Just as in the proof of Theorem 4.2, we have only to consider the case where $u$ is not a constant in $\Omega$. Our proof is based on a reduction to absurdity.

We may assume, to the contrary, that there exists a boundary point $x_{0}^{\prime}$ such that

$$
\left\{\begin{array}{l}
u\left(x_{0}^{\prime}\right)=\max _{x \in \bar{\Omega}} u(x)>0, \\
u\left(x_{0}^{\prime}+z\right)<u\left(x_{0}^{\prime}\right) \text { for all } x_{0}^{\prime}+z \in \Omega .
\end{array}\right.
$$

Then, by applying the Hopf boundary point lemma (Lemma 6.1) we obtain that

$$
\frac{\partial u}{\partial \mathbf{n}}\left(x_{0}^{\prime}\right)<0
$$

Moreover, since we have the assertions

$$
\begin{aligned}
& u\left(x_{0}^{\prime}+z\right)-u\left(x_{0}^{\prime}\right)<0 \text { for all } x_{0}^{\prime}+z \in \Omega, \\
& J(x, z) \geq 0 \quad \text { almost everywhere in } \mathbf{R}^{n} \times \mathbf{R}^{n},
\end{aligned}
$$

it follows that

$$
\begin{aligned}
0 & =L u\left(x_{0}^{\prime}\right)=\Lambda u\left(x_{0}^{\prime}\right)+T u\left(x_{0}^{\prime}\right) \\
& =\mu\left(x_{0}^{\prime}\right) \frac{\partial u}{\partial \mathbf{n}}\left(x_{0}^{\prime}\right)+\gamma\left(x_{0}^{\prime}\right) u\left(x_{0}^{\prime}\right)+\int_{\left\{x_{0}^{\prime}+z \in \Omega\right\}}\left(u\left(x_{0}^{\prime}+z\right)-u\left(x_{0}^{\prime}\right)\right) J\left(x_{0}^{\prime}, z\right) \nu(d z) .
\end{aligned}
$$

However, by condition (5.18) we obtain from assertions (5.20) and (5.21) that

$$
\begin{aligned}
& L u\left(x_{0}^{\prime}\right) \\
= & \mu\left(x_{0}^{\prime}\right) \frac{\partial u}{\partial \mathbf{n}}\left(x_{0}^{\prime}\right)+\gamma\left(x_{0}^{\prime}\right) u\left(x_{0}^{\prime}\right)+\int_{\left\{x_{0}^{\prime}+z \in \Omega\right\}}\left(u\left(x_{0}^{\prime}+z\right)-u\left(x_{0}^{\prime}\right)\right) J\left(x_{0}^{\prime}, z\right) \nu(d z) \\
< & 0 .
\end{aligned}
$$

This contradicts formula (5.22).

The proof of Theorem 5.3 is complete.

Remark 5.1 Intuitively, the transversality condition (5.19) implies that one of the reflection and absorption phenomena and the inward jump phenomenon from the boundary occurs at every point $x^{\prime} \in \partial \Omega$ (see [32]).

\section{The maximum principle for elliptic Waldenfels operators in Sobolev spaces}

In the last section, we prove various maximum principles for second-order, elliptic Waldenfels integro-differential operators with discontinuous coefficients such as the weak and strong maximum principles (Theorems 6.1 and 6.2) and the Hopf boundary point lemma (Lemma 6.1) in the framework of $L^{p}$ Sobolev spaces which play an essential role throughout the paper. The results here are adapted from Bony [5], [6], Bony-Courrège-Priouret [7], Garroni-Menaldi [12], Troianiello [38] and also Taira [32] and [34]. 
Let $\Omega$ be a bounded domain in Euclidean space $\mathbf{R}^{n}, n \geq 2$, with boundary $\partial \Omega$ of class $C^{1,1}$. We consider a second-order, elliptic Waldenfels integrodifferential operator $W$ with real $L^{\infty}$ coefficients such that

$$
W u(x)=A u(x)+S u(x), \quad x \in \Omega,
$$

where

$$
A u(x)=\sum_{i, j=1}^{n} a^{i j}(x) \frac{\partial^{2} u}{\partial x_{i} \partial x_{j}}(x)+\sum_{i=1}^{n} b^{i}(x) \frac{\partial u}{\partial x_{i}}(x)+c(x) u(x)
$$

and

$$
S u(x)=\int_{\mathbf{R}^{n} \backslash\{0\}}\left(u(x+z)-u(x)-\sum_{j=1}^{n} z_{j} \frac{\partial u}{\partial x_{j}}(x)\right) K(x, z) \mu(d z) .
$$

More precisely, we assume that the coefficients $a^{i j}(x), b^{i}(x)$ and $c(x)$ of the differential operator $A$ satisfy the following three conditions (1), (2) and (3):

(1) $a^{i j}(x) \in L^{\infty}(\Omega), a^{i j}(x)=a^{j i}(x)$ for all $1 \leq i, j \leq n$ and for almost all $x \in \Omega$ and there exist a constant $\lambda>0$ such that

$$
\frac{1}{\lambda}|\xi|^{2} \leq \sum_{i, j=1}^{n} a^{i j}(x) \xi_{i} \xi_{j} \leq \lambda|\xi|^{2}
$$

for almost all $x \in \Omega$ and all $\xi \in \mathbf{R}^{n}$.

(2) $b^{i}(x) \in L^{\infty}(\Omega)$ for all $1 \leq i \leq n$.

(3) $c(x) \in L^{\infty}(\Omega)$ and $c(x) \leq 0$ for almost all $x \in \Omega$.

(4) $K \in L^{\infty}\left(\mathbf{R}^{n} \times \mathbf{R}^{n}\right)$ with $K(x, y) \geq 0$ almost everywhere in $\mathbf{R}^{n} \times \mathbf{R}^{n}$, and it satisfies the growth condition (1.4) for some $0<\theta_{0}<1$ and condition (1.5).

(5) $\mu(d z)$ is a Radon measure on $\mathbf{R}^{n} \backslash\{0\}$ that has a density with respect to the Lebesgue measure $d z$ on $\mathbf{R}^{n}$, and it satisfies the moment condition (1.6).

6.1 The weak maximum principle

The purpose of this subsection is to formulate a variant of the weak maximum principle in the framework of $L^{p}$ Sobolev spaces, essentially due to Bony (cf. [5, Théorème 2], [13, Section 9.1, Theorem 9.1], [38, Chapter 3, Lemma 3.25]):

Theorem 6.1 (the weak maximum principle) Assume that a function $u$ in the Sobolev space $W^{2, p}(\Omega)$ with $n<p<\infty$ satisfies the condition

$$
W u(x) \geq 0 \text { for almost all } x \in \Omega \text {. }
$$

Then we have the inequality

$$
\max _{\bar{\Omega}} u \leq \max _{\partial \Omega} u^{+}
$$


where

$$
u^{+}(x)=\max \{u(x), 0\} \quad \text { for } x \in \bar{\Omega} .
$$

A detailed proof of Theorem 6.1 is given in Taira [34, Theorem 8.1].

\subsection{Hopf's boundary point lemma}

In this subsection, we study the inward normal derivative $\partial u / \partial \mathbf{n}\left(x_{0}^{\prime}\right)$ at a boundary point $x_{0}^{\prime}$ where the function $u(x)$ takes its non-negative maximum.

The Hopf boundary point lemma reads as follows (cf. [7, Théorème VIII], [12, Theorem 3.1.5]):

Lemma 6.1 (Hopf) Assume that a function $u \in W^{2, p}(\Omega)$, with $n<p<\infty$, satisfies the condition

$$
W u(x) \geq 0 \quad \text { for almost all } x \in \Omega .
$$

If $u(x)$ attains a non-negative, strict local maximum at a point $x_{0}^{\prime}$ of $\partial \Omega$, then we have the inequality

$$
\frac{\partial u}{\partial \mathbf{n}}\left(x_{0}^{\prime}\right)<0
$$

where $\mathbf{n}=\left(n_{1}, n_{2}, \ldots, n_{n}\right)$ is the unit inward normal to the boundary $\partial \Omega$.

Proof By Theorem 6.1 (or [34, Theorem 8.1]), it suffices to consider the case

$$
\left\{\begin{array}{l}
u\left(x_{0}^{\prime}\right)=m:=\max _{x \in \bar{\Omega}} u(x) \geq 0, \\
u(y)<u\left(x_{0}^{\prime}\right) \text { for all } y \in \Omega .
\end{array}\right.
$$

The proof of inequality (6.3) is divided into three steps.

Step 1: By condition (6.4), we can find an open ball $B(y, r)$ contained in the domain $\Omega$, centered at $y$, such that

(a) The point $x_{0}^{\prime}$ is on the boundary $S(y, r)=\{z \in \Omega:|z-y|=r\}$ of $B(y, r)$;

(b) $\mathbf{n}=s\left(y-x_{0}^{\prime}\right)$ for some $s>0$.

Step 2: Near the boundary point $x_{0}^{\prime}$, we introduce local coordinate systems $\left(x^{\prime}, x_{n}\right)$ such that $x^{\prime}=\left(x_{1}, x_{2}, \ldots, x_{n-1}\right)$ give local coordinates for the boundary $\partial \Omega$ and that

$$
\begin{aligned}
& \Omega=\left\{\left(x^{\prime}, x_{n}\right): x_{n}>0\right\}, \quad \partial \Omega=\left\{\left(x^{\prime}, x_{n}\right): x_{n}=0\right\}, \\
& x_{0}^{\prime}=(0, \ldots, 0,0) \in \partial \Omega, \quad y=(0, \ldots, 0, r) \in \Omega, \quad\left|x_{0}^{\prime}-y\right|=r .
\end{aligned}
$$

Substep 2-1: Now we introduce a function $v(x)$ by the formula

$$
v(x)=v\left(x^{\prime}, x_{n}\right)=\exp \left[-\gamma|x-y|^{2}\right]-\exp \left[-\gamma r^{2}\right],
$$

where $\gamma$ is a positive constant to be chosen later on. Then it is easy to see that

$$
A v(x)=\sum_{i, j=1}^{n} a^{i j}(x) \frac{\partial^{2} v}{\partial x_{i} \partial x_{j}}(x)+\sum_{i=1}^{n} b^{i}(x) \frac{\partial v}{\partial x_{i}}(x)+c(x) v(x)
$$




$$
\begin{aligned}
= & \exp \left[-\gamma|x-y|^{2}\right] \times\left[4 \gamma^{2}\left(\sum_{i, j=1}^{n} a^{i j}(x)\left(x_{i}-y_{i}\right)\left(x_{j}-y_{j}\right)\right)\right. \\
& \left.-2 \gamma\left(\sum_{i=1}^{n}\left(a^{i i}(x)+b^{i}(x)\left(x_{i}-y_{i}\right)\right)\right)\right]+c(x) v(x) .
\end{aligned}
$$

However, we have, by formula (6.5) and condition (6.1),

$$
v(x) \leq \exp \left[-\gamma|x-y|^{2}\right] \quad \text { for all } x \in \Omega,
$$

and

$$
\sum_{i, j=1}^{n} a^{i j}(x)\left(x_{i}-y_{i}\right)\left(x_{j}-y_{j}\right) \geq \frac{1}{\lambda}|x-y|^{2} \quad \text { for almost all } x \in \Omega \text {. }
$$

Hence, it follows from formula (6.6) that

$$
\begin{aligned}
A v(x) \geq & \exp \left[-\gamma|x-y|^{2}\right] \\
& \times\left[\frac{4 \gamma^{2}}{\lambda}|x-y|^{2}-2 \gamma\left(\sum_{i=1}^{n}\left(a^{i i}(x)+\left|b^{i}(x)\right|\left|x_{i}-y_{i}\right|\right)+|c(x)|\right)\right]
\end{aligned}
$$

for almost all $x \in \Omega$.

Moreover, for any $\rho>0$, we can choose a constant $\gamma=\gamma(\rho)>0$ so large that we have, for $\rho<|x-y|<r$,

$$
\begin{aligned}
& \frac{4 \gamma^{2}}{\lambda}|x-y|^{2}-2 \gamma\left(\sum_{i=1}^{n}\left(a^{i i}(x)+\left|b^{i}(x)\right|\left|x_{i}-y_{i}\right|\right)+|c(x)|\right) \\
\geq & \frac{4 \rho^{2}}{\lambda} \gamma^{2}-C \gamma,
\end{aligned}
$$

where

$$
\begin{aligned}
& C=2\left(\sum_{i=1}^{n}\left(\left\|a^{i i}\right\|_{L^{\infty}(\Omega)}+r\left\|b^{i}\right\|_{L^{\infty}(\Omega)}\right)+\|c\|_{L^{\infty}(\Omega)}\right), \\
& r=\left|x_{0}^{\prime}-y\right| .
\end{aligned}
$$

Therefore, by combining inequalities (6.7) and (6.8) we obtain that, for any $\rho>0$ there exists a constant $\gamma=\gamma(\rho)>0$ such that

$$
\begin{aligned}
A v(x) \geq & \left(\frac{4 \rho^{2}}{\lambda} \gamma^{2}-C \gamma\right) \exp \left[-\gamma|x-y|^{2}\right] \\
& \quad \text { for almost all } x \in \Gamma_{\rho r}:=\{z \in \Omega: \rho<|z-y|<r\} .
\end{aligned}
$$

Substep 2-2: On the other hand, we can obtain an $L^{\infty}$-version of Theorem 3.2 as follows: 
Lemma 6.2 For every $\varepsilon>0$, there exists a constant $C(\varepsilon)>0$ such that we have, for all $u \in C^{2}(\bar{\Omega})$,

$$
\|S u\|_{L^{\infty}(\Omega)} \leq \frac{1}{2} \sigma(\varepsilon)\|K\|_{\infty}\left\|\nabla^{2} u\right\|_{L^{\infty}(\Omega)}+C(\varepsilon)\|K\|_{\infty}\|\nabla u\|_{L^{\infty}(\Omega)} .
$$

Here

$$
\sigma(\varepsilon):=\int_{\{0<|z| \leq \varepsilon\}}|z|^{2} \mu(d z)
$$

Proof For each $\varepsilon>0$, we decompose the integral term $S u(x)$ into the two terms $S_{\varepsilon}^{(1)} u$ and $S_{\varepsilon}^{(2)} u$ as follows:

$$
S u(x)=S_{\varepsilon}^{(1)} u(x)+S_{\varepsilon}^{(2)} u(x) .
$$

Here:

$$
\begin{aligned}
S_{\varepsilon}^{(1)} u(x) & :=\int_{\{0<|z| \leq \varepsilon\}}\left(u(x+z)-u(x)-\sum_{j=1}^{n} z_{j} \frac{\partial u}{\partial x_{j}}(x)\right) K(x, z) \mu(d z) \\
& =\int_{0}^{1}(1-t) d t \int_{\{0<|z| \leq \varepsilon\}} z \cdot \nabla^{2} u(x+t z) z K(x, z) \mu(d z)
\end{aligned}
$$

and

$$
S_{\varepsilon}^{(2)} u(x):=\int_{\{|z|>\varepsilon\}}(u(x+z)-u(x)-z \cdot \nabla u) \mu(d z) .
$$

(1) First, we have the inequality

$$
\begin{aligned}
\left\|S_{\varepsilon}^{(1)} u\right\|_{L^{p}(\Omega)} & \leq \frac{1}{2}\|K\|_{\infty}\left(\int_{\{0<|z| \leq \varepsilon\}}|z|^{2} \mu(d z)\right)\left\|\nabla^{2} u\right\|_{L^{\infty}(\Omega)} \\
& =\frac{1}{2} \sigma(\varepsilon)\|K\|_{\infty}\left\|\nabla^{2} u\right\|_{L^{\infty}(\Omega)} .
\end{aligned}
$$

By condition (1.6), it follows from an application of Lebesgue's dominated convergence theorem that

$$
\lim _{\varepsilon \downarrow 0} \sigma(\varepsilon)=0 .
$$

(2) Secondly, we rewrite the term $S_{\varepsilon}^{(2)} u$ in the form

$$
\begin{aligned}
S_{\varepsilon}^{(2)} u(x)= & \int_{\{|z|>\varepsilon\}} K(x, z)(u(x+z)-u(x)) \mu(d z) \\
& +\sum_{j=1}^{n} \int_{\{|z|>\varepsilon\}} K(x, z) z_{j} \frac{\partial u}{\partial x_{j}}(x) \mu(d z) \\
:= & A(x)+B(x) .
\end{aligned}
$$

Then, by using condition (1.6) we can estimate the term $B(x)$ as follows:

$$
|B(x)| \leq \int_{\{|z|>\varepsilon\}} K(x, z)|z| \cdot \sum_{j=1}^{n}\left|\frac{\partial u}{\partial x_{j}}(x)\right| \mu(d z)
$$




$$
\begin{aligned}
& \leq\|K\|_{\infty}\left(\int_{\{|z|>\varepsilon\}}|z| \mu(d z)\right)\|\nabla u\|_{L^{\infty}(\Omega)} \\
& =\delta(\varepsilon)\|K\|_{\infty}\|\nabla u\|_{L^{\infty}(\Omega)} \quad \text { for all } x \in \Omega,
\end{aligned}
$$

where

$$
\delta(\varepsilon):=\int_{\{|z|>\varepsilon\}}|z| \mu(d z) .
$$

However, the term $\delta(\varepsilon)$ can be estimated as follows:

$$
\begin{aligned}
\delta(\varepsilon) & =\int_{\{|z|>1\}}|z| \mu(d z)+\int_{\{\varepsilon<|z| \leq 1\}}|z| \mu(d z) \\
& \leq \int_{\{|z|>1\}}|z| \mu(d z)+\frac{1}{\varepsilon} \int_{\{\varepsilon<|z| \leq 1\}}|z|^{2} \mu(d z) \\
& \leq \int_{\{|z|>1\}}|z| \mu(d z)+\frac{1}{\varepsilon} \int_{\{0<|z| \leq 1\}}|z|^{2} \mu(d z) \\
& =C_{2}+\frac{C_{1}}{\varepsilon} .
\end{aligned}
$$

Hence we obtain the inequality

$$
\|B\|_{L^{\infty}(\Omega)} \leq\left(\frac{C_{1}}{\varepsilon}+C_{2}\right)\|K\|_{\infty}\|\nabla u\|_{L^{\infty}(\Omega)} .
$$

On the other hand, by Morrey's imbedding theorem (see [13, Theorem 7.17]) we can find a constant $C>0$ such that

$$
|u(x+z)-u(x)| \leq C|z|^{1-n / p}\|\nabla u\|_{L^{p}(\Omega)} .
$$

Hence, it follows from inequality (6.13) that

$$
\begin{aligned}
& |A(x)| \leq \int_{\{|z|>\varepsilon\}} K(x, z)(u(x+z)-u(x)) \mu(d z) \\
& \leq C\|K\|_{\infty} \int_{\{|z|>\varepsilon\}}|z|^{1-n / p} \mu(d z) \cdot\|\nabla u\|_{L^{p}(\Omega)} \\
& =C\|K\|_{\infty} \int_{\{|z|>\varepsilon\}}|z| \cdot \frac{1}{|z|^{n / p}} \mu(d z)\|\nabla u\|_{L^{p}(\Omega)} \\
& \leq \frac{C\|K\|_{\infty}}{\varepsilon^{n / p}}\left(\int_{\{|z|>\varepsilon\}}|z| \mu(d z)\right)\|\nabla u\|_{L^{p}(\Omega)} \\
& =\delta(\varepsilon) \frac{C\|K\|_{\infty}}{\varepsilon^{n / p}}\|\nabla u\|_{L^{p}(\Omega)} \leq\left(\frac{C_{1}}{\varepsilon}+C_{2}\right) \frac{C\|K\|_{\infty}}{\varepsilon^{n / p}}\|\nabla u\|_{L^{p}(\Omega)} \\
& =C_{\varepsilon}\|K\|_{\infty}\|\nabla u\|_{L^{p}(\Omega)} \quad \text { for all } x \in \Omega \text {, }
\end{aligned}
$$

where

$$
C_{\varepsilon}:=\frac{C}{\varepsilon^{n / p}}\left(\frac{C_{1}}{\varepsilon}+C_{2}\right) .
$$


Thus we have the estimate

$$
\begin{aligned}
\|A\|_{L^{\infty}(\Omega)} & \leq\left(\frac{C_{1}}{\varepsilon}+C_{2}\right) \frac{C\|K\|_{\infty}}{\varepsilon^{n / p}}\|\nabla u\|_{L^{p}(\Omega)} \\
& =C_{\varepsilon}\|K\|_{\infty}\|\nabla u\|_{L^{p}(\Omega)} \\
& \leq C_{\varepsilon}\|K\|_{\infty} C_{3}|\Omega|^{1 / p}\|\nabla u\|_{L^{\infty}(\Omega)} \quad \text { for some constant } C_{3}>0
\end{aligned}
$$

where $|\Omega|$ is the volume of the domain $\Omega$.

Therefore, we obtain from estimates (6.14) and (6.15) that

$$
\left\|S_{\varepsilon}^{(2)} u\right\|_{L^{\infty}(\Omega)} \leq\|A\|_{L^{\infty}(\Omega)}+\|B\|_{L^{\infty}(\Omega)} \leq C(\varepsilon)\|K\|_{\infty}\|\nabla u\|_{L^{\infty}(\Omega)},
$$

where

$$
C(\varepsilon):=\left(\frac{C_{1}}{\varepsilon}+C_{2}\right)\left(1+\frac{C C_{3}}{\varepsilon^{n / p}}|\Omega|^{1 / p}\right) .
$$

(3) The desired estimate (6.10) follows by combining estimates (6.11) and (6.16):

$$
\begin{aligned}
\|S u\|_{L^{\infty}(\Omega)} & \leq\left\|S_{\varepsilon}^{(1)} u\right\|_{L^{\infty}(\Omega)}+\left\|S_{\varepsilon}^{(2)} u\right\|_{L^{\infty}(\Omega)} \\
& \leq \frac{1}{2} \sigma(\varepsilon)\|K\|_{\infty}\left\|\nabla^{2} u\right\|_{L^{\infty}(\Omega)}+C(\varepsilon)\|K\|_{\infty}\|\nabla u\|_{L^{\infty}(\Omega)} .
\end{aligned}
$$

The proof of Lemma 6.2 is complete.

By combining inequality (6.10) (for the function $v$ defined by formula (6.5)) with assertion (6.12), we can find that, for every small $\eta>0$ there exists a constant $C_{\eta}>0$ such that

$$
\begin{aligned}
& |S v(x)| \leq\left(\eta \gamma^{2}+C_{\eta} \gamma\right) \exp \left[-\gamma|x-y|^{2}\right] \\
& \quad \text { for almost all } x \in \Gamma_{\rho r}:=\{z \in \Omega: \rho<|z-y|<r\} .
\end{aligned}
$$

Therefore, by taking

$$
\begin{aligned}
& \eta:=\frac{2 \rho^{2}}{\lambda}, \\
& \gamma>\frac{\lambda}{2 \rho^{2}}\left(C_{\eta}+C\right),
\end{aligned}
$$

we obtain from inequalities (6.9) and (6.17) that

$$
\begin{aligned}
W v(x) & =A v(x)+S v(x) \geq A v(x)-|S v(x)| \\
& \geq\left(\frac{2 \rho^{2}}{\lambda} \gamma^{2}-\left(C+C_{\eta}\right) \gamma\right) \exp \left[-\gamma|x-y|^{2}\right] \\
& =\frac{2 \rho^{2}}{\lambda} \gamma\left(\gamma-\frac{\lambda\left(C+C_{\eta}\right)}{2 \rho^{2}}\right) \exp \left[-\gamma|x-y|^{2}\right] \\
& >0 \quad \text { for almost all } x \in \Gamma_{\rho r}:=\{z \in \Omega: \rho<|z-y|<r\} .
\end{aligned}
$$


Step 3: Without loss of generality, we may assume that

$$
u(x)<u\left(x_{0}\right) \text { in } B(y, r) .
$$

If $\varepsilon>0$, we let

$$
w(x):=u(x)-u\left(x_{0}\right)+\varepsilon v(x) .
$$

(a) First, we have, by condition (6.19),

$$
w(x)=u(x)-u\left(x_{0}\right)+\varepsilon v(x) \leq 0 \quad \text { on } S(y, \rho)=\{z \in \Omega:|z-y|=\rho\},
$$

if $\varepsilon>0$ is chosen sufficiently small.

(b) Secondly, it follows that

$$
w(x)=u(x)-u\left(x_{0}\right)+\varepsilon v(x) \leq 0 \quad \text { on } S(y, r)=\{z \in \Omega:|z-y|=r\},
$$

since $v(x)=0$ on $S(y, r)$.

Hence we have, by assertions (a) and (b),

$$
w(x) \leq 0 \quad \text { on } \partial \Gamma_{\rho r}=S(y, \rho) \cup S(y, r) .
$$

On the other hand, by inequalities (6.2) and (6.18) it follows that

$$
\begin{aligned}
W w(x) & =W u(x)+\varepsilon W v(x)-u\left(x_{0}\right)(W 1)(x) \geq \varepsilon W v(x)-c(x) u\left(x_{0}\right) \\
& >-c(x) u\left(x_{0}\right) \\
& \geq 0 \quad \text { for almost all } x \in \Gamma_{\rho r}=B(y, r) \backslash \overline{B(y, \rho)} .
\end{aligned}
$$

Therefore, by applying the weak maximum principle (Theorem 6.1) with $\Omega:=\Gamma_{\rho r}$ we obtain that

$$
w(x) \leq 0 \quad \text { in } \Gamma_{\rho r}=B(y, r) \backslash \overline{B(y, \rho)} .
$$

(c) On the other hand, we have the formula

$$
w\left(x_{0}\right)=\varepsilon v\left(x_{0}\right)=0 .
$$

Therefore, it follows from assertions (6.20) and (6.21) that

$$
\frac{\partial w}{\partial \mathbf{n}}\left(x_{0}^{\prime}\right)=\frac{\partial u}{\partial \mathbf{n}}\left(x_{0}^{\prime}\right)+\varepsilon \frac{\partial v}{\partial \mathbf{n}}\left(x_{0}^{\prime}\right) \leq 0 .
$$

However, we have, by formula (6.5),

$$
\frac{\partial v}{\partial \mathbf{n}}\left(x_{0}^{\prime}\right)=2 \gamma r e^{-\gamma r^{2}}>0
$$

Summing up, we obtain from inequalities (6.22) and (6.23) that

$$
\frac{\partial u}{\partial \mathbf{n}}\left(x_{0}^{\prime}\right) \leq-\varepsilon \frac{\partial v}{\partial \mathbf{n}}\left(x_{0}^{\prime}\right)=-2 \varepsilon \gamma r e^{-\gamma r^{2}}<0 .
$$

Now the proof of Lemma 6.1 is complete. 
6.3 The strong maximum principle

Finally, we can prove the following strong maximum principle for the operator $A$ (cf. [5, Théorème 2], [7, Théorème VII], [12, Theorem 3.1.15]):

Theorem 6.2 (the strong maximum principle) Assume that a function $u \in W^{2, p}(\Omega)$, with $n<p<\infty$, satisfies the condition

$$
W u(x) \geq 0 \quad \text { for almost all } x \in \Omega \text {. }
$$

If $u(x)$ attains a non-negative maximum at an interior point of $\Omega$, then it is a (non-negative) constant function.

Proof Our proof is based on a reduction to absurdity. We let

$$
\begin{aligned}
& m:=\max _{x \in \bar{\Omega}} u(x) \geq 0, \\
& \mathcal{S}:=\{x \in \Omega: u(x)=m\},
\end{aligned}
$$

and assume, to the contrary, that

$$
\mathcal{S} \varsubsetneqq \Omega .
$$

Since $\mathcal{S}$ is closed in $\Omega$, we can find a point $x_{0}$ of $\mathcal{S}$ and an open ball $B(y, R)$ contained in the set $\Omega \backslash \mathcal{S}$, centered at $y$, such that

(a) $B(y, R) \subset \Omega \backslash \mathcal{S}$

(b) $x_{0}$ is on the boundary $S(y, R)=\{z \in \Omega:|z-y|=R\}$ of $B(y, R)$.

By applying the Hopf boundary point lemma (Lemma 6.1) with $\Omega:=$ $B(y, R)$, we obtain that

$$
\sum_{i=1}^{n} n_{i} \frac{\partial u}{\partial x_{i}}\left(x_{0}\right)<0
$$

where

$$
\mathbf{n}=\left(n_{1}, n_{2}, \ldots, n_{n}\right)=\frac{y-x_{0}}{\left|y-x_{0}\right|} .
$$

However, since $u\left(x_{0}\right)=m$ for some interior point $x_{0} \in \Omega$, it follows that

$$
\frac{\partial u}{\partial x_{i}}\left(x_{0}\right)=0, \quad 1 \leq i \leq n
$$

Hence we have the assertion

$$
\sum_{i=1}^{n} n_{i} \frac{\partial u}{\partial x_{i}}\left(x_{0}\right)=0 .
$$

This contradicts inequality (6.24).

The proof of Theorem 6.2 is complete. 


\section{References}

1. R. A. Adams and J. J. F. Fournier, Sobolev spaces, second edition. Pure and Applied Mathematics, Elsevier/Academic Press, Amsterdam (2003)

2. R. F. Anderson, Diffusions with second order boundary conditions I, Indiana Univ. Math. J. 25, 367-397 (1976)

3. R. F. Anderson, Diffusions with second order boundary conditions II, Indiana Univ. Math. J. 25, 403-441 (1976)

4. J. Bergh and J. Löfström, Interpolation spaces, an introduction. Springer-Verlag, Berlin Heidelberg New York (1976)

5. J.-M. Bony, Principe du maximum dans les espaces de Sobolev, C. R. Acad. Sci. Paris Sér. A-B 265, A333-A336 (1967)

6. J.-M. Bony, Problème de Dirichlet et semi-groupes fortement fellérien associés à un opérateur intégro-differentiel, C. R. Acad. Sci. Paris Sér. A-B 265, A361-A364 (1967)

7. J.-M. Bony, P. Courrège, P. Priouret, Semi-groupes de Feller sur une variété à bord compacte et problèmes aux limites intégro-différentiels du second ordre donnant lieu au principe du maximum, Ann. Inst. Fourier (Grenoble) 19, 369-521 (1968)

8. A. P. Calderón, Intermediate spaces and interpolation, the complex method. Studia Math. 24, 113-190 (1964)

9. P. Cattiaux, Hypoellipticité et hypoellipticité partielle pour les diffusions avec une condition frontière, Ann. Inst. H. Poincaré Probab. Statist. 22, 67-112 (1986)

10. M. Cwikel and N. J. Kalton, Interpolation of compact operators by the methods of Calderón and Gustavsson-Peetre, Proc. Edinburgh Math. Soc. (2) 38, 261-276 (1995)

11. G. B. Folland, Real analysis. Modern techniques and their applications. second edition. Pure and Applied Mathematics (New York). A Wiley-Interscience Publication. John Wiley \& Sons, Inc., New York (1999)

12. M. G. Garroni and J. L. Menaldi, Second order elliptic integro-differential problems, Research Notes in Mathematics Series no. 430, Chapman \& Hall/CRC, Boca Raton London New York Washington, D. C. (2002)

13. D. Gilbarg and N.S. Trudinger, Elliptic partial differential equations of second order, 1998 edition, Springer-Verlag, New York Berlin Heidelberg Tokyo (1998)

14. F. Gimbert and P.-L. Lions, Existence and regularity results for solutions of secondorder, elliptic, integrodifferential operators, Ricerche di Matematica 33, 315-358 (1984)

15. I. C. Gohberg and M. G, Kreı̆n, The basic propositions on defect numbers, root numbers and indices of linear operators, Uspehi Mat. Nauk. 12, 43-118 (1957) (Russian); English translation in Amer. Math. Soc. Transl. (2) 13, 185-264 (1960)

16. N. Ikeda and S. Watanabe, Stochastic differential equations and diffusion processes, second edition. North-Holland Publishing Co., Amsterdam; Kodansha Ltd., Tokyo (1989)

17. Y. Ishikawa, Stochastic calculus of variations for jump processes, second edition. De Gruyter Studies in Mathematics, vol. 54, De Gruyter, Berlin Boston (2016)

18. T. Komatsu, Markov processes associated with certain integro-differential operators. Osaka J. Math. 10, 271-303 (1973)

19. S. Lenhart, Integro-differential operators associated with diffusion processes with jumps. Appl. Math. Optim. 9, 177-191 (1982/83)

20. J. Malý, and W. P. Ziemer, Fine regularity of solutions of elliptic partial differential equations, American Mathematical Society, Providence, Rhode Island (1997)

21. J. Peetre, Rectification à l'article "Une caractérisation des opérateurs différentiels" Math. Scand. 8, 116-120 (1960)

22. T. Runst, Degenerate boundary value problems in Sobolev spaces of fractional order, Vestnik RUDN, Ser. Mathematics 6, 173-181 (1999)

23. T. Runst and W. Sickel, Sobolev spaces of fractional order, Nemytskij operators, and nonlinear partial differential equations, Walter de Gruyter, Berlin New York (1996)

24. T. Runst and A. Youssfi, Boundary value problems for Waldenfels operators, Indiana Univ. Math. J. 54, 237-255 (2005)

25. K. Sato, Lévy processes and infinitely divisible distributions, revised edition, Cambridge Studies in Advanced Mathematics, vol. 68, Cambridge University Press, Cambridge (2013) 
26. M. Schechter, Principles of functional analysis, second edition. Graduate Studies in Mathematics, vol. 36. American Mathematical Society, Providence, Rhode Island (2002)

27. R. T. Seeley, Extension of $C^{\infty}$ functions defined in a half-space, Proc. Amer. Math. Soc. 15, 625-626 (1964)

28. E. M. Stein, Singular integrals and differentiability properties of functions, Princeton Mathematical Series, Princeton University Press, Princeton, New Jersey (1970)

29. D. W. Stroock, Diffusion processes associated with Lévy generators. Zeitschrift für Wahrscheinlichkeitstheorie und Verwandte Gebiete, 32, 209-244 (1975)

30. K. Taira, Boundary value problems for elliptic integro-differential equations, Math. Z. 222, 305-327 (1996)

31. K. Taira, Boundary value problems and Markov processes, second edition. Lecture Notes in Mathematics, no. 1499, Springer-Verlag, Berlin Heidelberg New York (2009)

32. K. Taira, Semigroups, boundary value problems and Markov processes, second edition. Springer Monographs in Mathematics. Springer-Verlag, Heidelberg (2014)

33. K. Taira, Analytic semigroups and semilinear initial-boundary value problems, second edition, London Mathematical Society Lecture Note Series, vol. 434, Cambridge University Press, Cambridge (2016)

34. K. Taira, Dirichlet problems with discontinuous coefficients and Feller semigroups. Rend. Circ. Mat. Palermo (2). Advance online publication. Doi:10.1007/s12215-019-00404-5

35. S. Takanobu and S. Watanabe, On the existence and uniqueness of diffusion processes with Wentzell's boundary conditions. J. Math. Kyoto Univ. 28, 71-80 (1988)

36. H. Triebel, Theory of function spaces, reprint of the 1983 edition. Monographs in Mathematics, Birkhäuser Verlag, Basel (1983)

37. H. Triebel, Theory of function spaces II, reprint of the 1992 edition. Monographs in Mathematics, Birkhäuser Verlag, Basel (1992)

38. G. M. Troianiello, Elliptic differential equations and obstacle problems, The University Series in Mathematics, Plenum Press, New York London (1987)

39. M. I. Višik, On general boundary problems for elliptic differential equations (Russian). Trudy Moskov. Mat. Obšč. 1, 187-246 (1952); English translation in Amer. Math. Soc. Transl. (2) 24, 107-172 (1963)

40. W. von Waldenfels, Positive Halbgruppen auf einem $n$-dimensionalen Torus, Arch. Math. 15, 191-203 (1964)

41. A. D. Wentzell (Ventcel'), On boundary conditions for multi-dimensional diffusion processes, Theor. Probability Appl. 4, 164-177 (1959)

42. J. Wloka, Partial differential equations, Cambridge University Press, Cambridge (1987)

43. K. Yosida, Functional analysis, reprint of the sixth (1980) edition, Classics in Mathematics. Springer-Verlag, Berlin Heidelberg New York (1995)

44. W. P. Ziemer, Weakly differentiable functions, Sobolev spaces and functions of bounded variation, Graduate Texts in Mathematics, vol. 120, Springer-Verlag, New York (1989) 